\title{
A Modified Effective Binary Diffusion Model
}

\author{
YOUXUE ZHANG ${ }^{1}$ \\ Division of Geological and Planetary Sciences, California Institure of Technology, Pasadena
}

\begin{abstract}
Uphill diffusion, the diffusion of a component up its own concentration gradient, has often been observed in experimental studies of diffusion in synthetic multicomponent systems and natural silicate melts. To apply experimental data to natural processes, it is necessary to quantify this effect. A modified effective binary diffusion model is developed in this paper. The diffusive flux of a component is assumed to be proportional to its activity gradient (instead of concentration gradient). The activity coefficient is related to its concentration at quasi-equilibrium distribution in a compositional continuum with the help of the two-liquid partition concept. The concentration at quasi-equilibrium distribution is approximately expressed as a simple function of $\mathrm{SiO}_{2}$ or $\mathrm{SiO}_{2}+\mathrm{Al}_{2} \mathrm{O}_{3}$ concentration. The diffusion of $\mathrm{SiO}_{2}$ or $\mathrm{SiO}_{2}+\mathrm{Al}_{2} \mathrm{O}_{3}$ is assumed to be effectively binary. With these assumptions, diffusion of a major or trace component can be described by two adjustable parameters, $\mathcal{D}$ (the intrinsic effective binary diffusivity) and $C_{f}$ (a parameter related to two-liquid partition coefficients). The simple model fits well the experimental uphill diffusion profiles produced by diffusive crystal dissolution into an andesitic melt using constant diffusivities when compositional variation across the whole profile is small. The parameters fit to the profiles are generally physically meaningful. The model is able to predict the direction of diffusion (occurrence or absence of uphill diffusion) in previous experiments and can be used to simulate the development of chemical concentration and isotopic ratio profiles during diffusion. When compositional dependence of diffusivities is allowed, the model may be applied to systems with greater compositional variation. This model can be tested in several ways and should be useful in studying diffusion in a variety of natural systems.
\end{abstract}

\section{INTRODUCTION}

Understanding of diffusion in natural silicate melts is essential to the quantitative description of kinetic processes in geology. The growth, dissolution, exsolution, and zonation of crystals [e.g., Albarede and Bottinga, 1972; Kirkpatrick, 1975, 1981; Dowty, 1980; Lasaga, 1981, 1982; Watson, 1982; Tsuchiyama and Takahashi, 1983; Harrison and Watsom, 1983, 1984; Donaldson, 1985, 1990; Kuo and Kirkpatrick, 1985; Tsuchiyama, 1985, Brearley and Scarfe, 1986; Duda and Shimizu, 1986; Ghiorso, 1987a; Ryerson and Watson, 1987; Zhang et al., 1989], the mixing, unmixing, zonation, and double diffusive convection of magmas [e.g., Hildreth, 1981; Trial and Spera, 1988; Spera et al., 1989; Baker, 1990], the partial melting of the mantle [e.g., McKenzie, 1984; Fisk, 1986], and the isotopic and chemical exchange in the mantle and in melts [e.g., Hofmann and Hart, 1978; Sneeringer et al., 1984; Baker, 1989; Lesher, 1990; van der Laan et al., 1990] are all controlled by or related to multicomponent diffusion. Uphill diffusion, the diffusion of a component against its own concentration gradient, is often observed in experimental studies of diffusion in multicomponent silicate melts [e.g., Sato, 1975; Watson, 1982; Donaldson, 1985; Fisk, 1986; Zhang et al., 1989; Lesher, 1990; van der Laan et al., 1990] and in rocks [e.g., Sato, 1975; Davidson et al., 1990]. Sato [1975] found alkali enrichment in diffusion coronas around quartz xenocrysts in andesite and basalt, and he conducted experiments to confirm that the enrichment was due to the uphill diffusion of alkalis toward silica-rich melt around dissolving quartz. Watson [1982] and Fisk [1986] also observed uphill diffusion of alkalis in their experiments. Donaldson [1985] noted $\mathrm{FeO}$ enrichment in the melt near olivine-melt interface during

\footnotetext{
${ }^{1}$ Now at Department of Geological Sciences, University of Michigan, Ann Arbor.
}

Copyright 1993 by the American Geophysical Union.

Paper number 93JB00422.

0148-0227/93/93JB-00422\$05.00 olivine dissolution experiments. Zhang et al. [1989] found that a large number of major and trace components may diffuse uphill in natural silicate melts. For example, during diffusive olivine dissolution into an andesitic melt, $\mathrm{FeO}, \mathrm{CaO}, \mathrm{TiO}_{2}, \mathrm{~V}_{2} \mathrm{O}_{5}, \mathrm{Ce}_{2} \mathrm{O}_{3}$, $\mathrm{Y}_{2} \mathrm{O}_{3}, \mathrm{Sc}_{2} \mathrm{O}_{3}$, and $\mathrm{ZrO}_{2}$ diffuse uphill toward less polymerized melt, and alkalis $\left(\mathrm{Na}_{2} \mathrm{O}, \mathrm{K}_{2} \mathrm{O}\right.$, and $\left.\mathrm{Rb}_{2} \mathrm{O}\right)$ diffuse uphill toward more polymerized melt. Lesher [1990] and van der Laan et al. [1990] observed uphill diffusion of $\mathrm{Sr}, \mathrm{Nd}, \mathrm{CaO}$, and $\mathrm{Na}_{2} \mathrm{O}$ in diffusion couple experiments between basalt and rhyolite and between two rhyolites. On the other hand, Baker [1990] observed no uphill diffusion during similar diffusion couple experiments between dacite and rhyolite. At present, there is no practical model that can be used to predict whether uphill diffusion will occur in a given system and to quantitatively predict the concentration profiles. In other words, after decades of diffusion studies, there is still no way to predict the direction of diffusion in natural silicate melts, not to mention the detailed structure of a concentration profile. In order to predict diffusive behavior in natural silicate melts during magma evolution, it is first necessary to understand the occurrence and absence of uphill diffusion and to account for disparities in controlled experimental studies. This paper presents an approximate treatment of diffusion in multicomponent systems that can be applied to determine the direction of diffusion and to semiquantitatively predict uphill diffusion profiles.

\section{THEORETICAL BACKGROUND}

There are two approaches to uphill diffusion in a multicomponent system. One approximate explanation [Sato, 1975; Watson, 1982] is to assume that the component in consideration always diffuses down its own activity gradient (i.e., down its chemical potential gradient) and that uphill diffusion (in terms of concentration) is a reflection that activity is not proportional to concentration. In other words, one may view uphill diffusion as a component trying to reach equilibrium "partition" along a compositional continuum of the silicate melt. This idea arises from experimental data on the partitioning behavior of elements between two immiscible silicate liquids 
[e.g., Watson, 1976; Ryerson and Hess, 1978]. Watson [1982] first suggested and described the relationship between two-liquid partition coefficients (as indicative of relative activity coefficients) and multicomponent diffusion behavior. When equilibrium is reached in such partitioning experiments, chemical potential (or activity) of a component is uniform, but its concentration is not. In general, alkalis tend to be enriched in more polymerized melts (melts enriched in $\mathrm{SiO}_{2}$ and/or $\mathrm{Al}_{2} \mathrm{O}_{3}$ ), and other components tend to be enriched in less polymerized melts. Since alkalis partition preferentially into more polymerized melt at uniform chemical potential, they may diffuse uphill against their concentration gradients (but still down their activity gradients) toward more polymerized melt. Since other components partition preferentially into less polymerized melt, they may diffuse uphill against their own concentration gradients toward less polymerized melt. This is an appealing explanation, but it has not been used to quantify diffusion profiles; the model presented in this paper is a quantitative model based on this explanation.

The other, more general, description is a phenomenological multicomponent diffusion treatment including cross-term diffusion coefficients. In this treatment, the diffusive flux of a component $i\left(J_{i}\right)$ in an $N$ component system is written as [De Groot and Mazur, 1962]

$$
J_{i}=-D_{i j} \nabla C_{i}-\sum_{j, j \neq i}^{N-1} D_{i j} \nabla C_{j}, \quad i=1,2, \ldots, N-1
$$

where the $N$ th component is defined as the dependent component, $C_{i}$ is the concentration of component $i$ (mass or mole per volume), $D_{i j}$ is the diffusivity of component $i$ due to its own concentration gradient, and $D_{i j}$ is the diffusivity of component $i$ due to a concentration gradient in component $j$ (the notation list explains symbols that appear twice or more in this paper). Diffusion in an $N$-component system is thus characterized by an $N-1$ by $N-1$ diffusivity matrix (hereafter referred as the $[D]$ matrix) in which $D_{i i}$ is called a diagonal term and $D_{i j}$ (when $i \neq j$ ) an off-diagonal term, or a cross-term diffusivity. Based on irreversible thermodynamics, all eigenvalues of a $[D]$ matrix must be real; they are all positive if the phase is stable, and one or more are negative if the phase is unstable with respect to spinodal decomposition [e.g., Kirkaldy and Young, 1987]. Uphill diffusion may occur in two cases. It may occur if the $[D]$ matrix has negative eigenvalues (spinodal decomposition). It may also occur even if all eigenvalues of the $[D]$ matrix are positive because the diffusive flux $J_{i}$ is not constrained by the sign of the first term in (1) (although there may be constraints relating offand on-diagonal diffusivities under certain conditions [e.g.. Cooper, 1965; Lasaga, 1979; Baker, 1992]). A component $i$ may diffuse uphill (i.e., $J_{i}$ is opposite to $-\nabla C_{i}$ ), especially when $\left|\nabla C_{i}\right|$ is small relative to $\left|\nabla C_{j}\right|$ such that the off-diagonal terms in (1) dominate.

This phenomenological treatment is general in that it should always work if each $D_{i j}$ in the diffusivity matrix is allowed to vary as a function of composition at constant $T$ and $P$ [Gupta and Cooper, 1971] and if negative diagonal $D_{i j}$ values and negative eigenvalues for the $[D]$ matrix are allowed. Indeed, this theory has been successfully applied to ternary systems (see review of Kirkaldy and Young [1987]). In practice, however, obtaining a [D] matrix from experimental data on a quatemary or more complicated system (most natural silicate melts consist of at least six major components, $\mathrm{SiO}_{2}, \mathrm{Al}_{2} \mathrm{O}_{3}, \mathrm{FeO}, \mathrm{MgO}, \mathrm{CaO}, \mathrm{Na}_{2} \mathrm{O}$ ) has proven difficult. For example, Zhang et al. [1989] attempted to obtain a $[D]$ matrix for natural andesitic melts from diffusion profiles produced by diffusive crystal dissolution but concluded that the cross-term diffusivities were too poorly constrained by the data to justify reporting a diffusivity matrix. Kubicki et al. [1990] gave multicomponent diffusivity matrices for a quatemary system $\left(\mathrm{SiO}_{2}-\mathrm{Al}_{2} \mathrm{O}_{3}-\mathrm{MgO}-\mathrm{CaO}\right.$ on the $\mathrm{An}-\mathrm{Di}$ join). However, these matrices have complex eigenvalues, which are in violation of basic constraints from irreversible thermodynamics. Trial and Spera [1990] reported a preliminary diffusivity matrix (which also has complex eigenvalues due to truncation errors in each element in the matrix); they also concluded that elements in the matrix were so poorly constrained that it cannot be applied at all to predict diffusion behavior in other systems even with the same bulk composition. Since obtaining diffusivity matrix from experimental data is difficult, Baker [1992] applied the model of Lasaga [1979] to calculate diffusivity matrices from tracer diffusivities. The matrices are then used to calculate concentration profiles in his dacite-rhyolite couples [Baker, 1990]. The results show mixed success and do not yet provide a reliable method for predicting chemical diffusion profiles.

The difficulty of obtaining multicomponent diffusivity matrices has led many workers to employ a concentration-based effective binary approach [Cooper, 1968; Hofmann, 1980]. In geological literature, almost all chemical diffusion data are reported on the basis of concentration-based effective binary diffusion [cf. Hofmann, 1980; Freer, 1981; Watson and Baker, 1991]. In this approach, the multicomponent system is treated as if it were binary between the component in consideration and the other combined component which includes all other components. The diffusive flux for component $i$ becomes

$$
J_{i}=-D_{i} \nabla C_{i}
$$

where $D_{i}$ is the concentration-based effective binary diffusivity for component $i$. This approximation is operationally feasible for monotonic diffusion profiles, but it does not work for components displaying uphill diffusion, because $D_{i}$ would vary from positive to infinity to negative in a single profile. At present, there is no way of predicting the direction of diffusion of a component in silicate melts in experimental charges or in natural magmas. Furthermore, concentration-based effective binary diffusivities extracted from experimental data seem overly dependent on melt composition even for monotonic profiles that are treatable by the method.

Even though treating multicomponent diffusion by (1) is completely general, it has a serious problem in that kinetic diffusive properties are lumped together with nonideal mixing properties of a solution (such as a melt). For example, negative interdiffusivities in binary systems and negative eigenvalues in diffusivity matrices for multicomponent systems during spinodal decomposition are a manifestation of nonideal mixing and not an intrinsic transport property [Kirkaldy and Young, 1987]. The lumping of kinetic diffusive properties with nonideal mixing properties makes the $[D]$ matrix defined in (1) overly sensitive to the bulk composition of the phase in nonideal systems. It would be preferable to separate kinetic properties (such as the diffusivity matrix) from thermodynamic properties, i.e., to separate crossterm diffusivities intrinsic to the transport process from those arising from nonideal mixing. Therefore it is preferable to write the diffusive flux in an $N$-component system according to Onsager's irreversible thermodynamics in terms of activity gradients as follows:

$$
J_{i}=-\mathcal{D}_{i i} \frac{\nabla a_{i}}{\gamma_{i}}-\sum_{j, j \star i}^{N-1} \mathcal{D}_{i j} \frac{\nabla a_{j}}{\gamma_{j}},
$$

where $a_{i}$ is the activity and $\gamma_{i}$ is the activity coefficient of component $i$ in the solution. $\mathcal{D}_{i j}$ is the activity-based diffusivity and will be referred as an intrinsic diffusivity [Carman, 1968] 
hereafter $\left(D_{i j}\right.$ will be referred as a concentration-based diffusivity). Note the difference between $\mathcal{D}_{i j}$ and $D_{i j}$, both in notation and in definition. The diffusivity matrix defined in (3) will be referred as the [D] matrix. Equation (3) is equivalent to and can be derived from irreversible thermodynamic expression for fluxes $\left(J_{i}=\Sigma_{i j} X_{j}\right.$, where $L_{i j}$ is the phenomenological coefficient and $\boldsymbol{X}_{j}$ is the thermodynamic force for diffusion) by letting $\mathcal{D}_{i j}=R T L_{i j} / C_{j}$ if $-\nabla \mu_{i}$ terms $(i=1,2, \ldots, N-1)$ are used as independent thermodynamic forces. The irreversible thermodynamic form $\left(J_{i}=\Sigma L_{i j} X_{j}\right)$ is not directly used because $L_{i j}$ is roughly proportional to $C_{j}$ and hence $\mathcal{D}_{i j}$ is less dependent on concentrations than $L_{i j}$ (cf., p. 15 of Kirkaldy and Young [1987]). Without the activity coefficient $\gamma\left(\gamma_{i}=a_{i} / C_{i}\right)$, (3) would be equivalent to replacing $C_{i}$ in (1) with $a_{i}$. The activity coefficients are included in (3) so that $\mathcal{D}_{i j}$ will equal $D_{i j}$ for cases with constant activity coefficients. The $[\mathcal{D}]$ matrix is expected to have real positive eigenvalues, and $[\mathcal{D}]$ should be more independent of concentrations than $[D]$ because the concentration dependence of mixing properties is excluded from [ $\mathcal{D}]$. For example, in binary systems the interdiffusivity $D$ varies from positive to negative during spinodal decomposition whereas $\mathcal{D}$ is always positive. In fact, spinodal decomposition can be simulated using a regular solution model and a constant $\mathcal{D}$.

The approach with (3) is theoretically appealing, but in practice, it is difficult to use because concentrations in a diffusion profile can be measured directly, while activities must be determined independently. Even if the $[\mathcal{D}]$ or $[D]$ matrix for major components can be obtained with sufficient accuracy to be applied to natural systems, it is unlikely in the near future that cross-term diffusivities for trace components due to concentration or activity gradients of major components could be resolved accurately enough to allow prediction of the diffusive behavior of the trace components. Hence it is useful to develop an approximate procedure for modeling the diffusion behavior for both major and trace components in complex systems that can be calibrated using limited experimental data and applied semiquantitatively to natural systems. In the sections that follow a modified effective binary diffusion treatment is presented in which activities are used instead of concentrations. The assumptions and formulation of the model are discussed first; then some features of and some simulation results based on the model are presented; afterward the model is applied to fit and predict diffusion profiles.

\section{A MODIFIED EFFECTIVE BINARY DIFFUSION MODEL}

As discussed earlier, there are insufficient data to achieve a perfect description of multicomponent diffusion through either (1) or (3) for complicated silicate melts. The concentration-based effective binary approach fails for components that diffuse uphill. As a compromise, a modified effective binary diffusion model is proposed. The basic approach is to approximate (3) by

$$
J_{i} \approx-\mathcal{D}_{i} \frac{\nabla a_{i}}{\gamma_{i}},
$$

for an $N$ component system where $i \neq N$. The flux of component $N$ (e.g., $\left.\mathrm{Al}_{2} \mathrm{O}_{3}\right)$ can be defined via mass balance $\left(\sum \alpha_{i} J_{i}=0\right.$ with $i$ $=1$ to $N$ where $\alpha_{i}$ terms are the normalization weights [De Groot and Mazur, 1962, p. 241]). The term $\mathcal{D}_{i}$ will be referred as an intrinsic effective binary diffusivity. Expressions similar to (4) have been employed or alluded to before [e.g., Lasaga, 1979; Watson, 1982]. Equation (4) holds exactly (i.e., equivalent to equation (3)) for binary systems and can be applied to simulate spinodal decomposition given a solution model (such as a regular solution model) to relate activity and concentration. In multicomponent systems, (4) lumps the cross-term diffusivities $\left(\mathcal{D}_{i j}\right.$ with $i \neq j$ ) into $\mathcal{D}_{i}$. Strictly speaking, intrinsic cross-term diffusivities $\left(\mathcal{D}_{i j}\right.$ with $i \neq j$ ) should not be lumped into (4) even if the diffusive flux is expressed as a function of activity gradient. Intrinsic uphill diffusion, i.e., diffusion of a component against its activity gradient, does occur in some systems (e.g., uphill diffusion in the $\mathrm{Cu}-\mathrm{Ag}-\mathrm{Au}$ metal system, which means intrinsic uphill diffusion because the system is near ideal [cf. Kirkaldy and Young, 1987; see also Spera and Trial, 1993]. Such a phenomenon cannot be treated by (4). Equation (4) also has the following limitations by analogy to the concentration-based effective binary approximation [Cooper, 1968]: (1) The activity gradients of all species must be in the same direction; and (2) either a steady state exists or there is no characteristic distance involved in the problem (i.e., infinite or semi-infinite diffusion). The approximations implicit in (4) undoubtedly cause some error, but modeling results to be shown later suggest that (1) such errors are not dominating for the systems studied; (2) this model does a better job than the concentration-based effective binary diffusion model in treating a variety of diffusion profiles; and (3) this model is able to predict the direction of diffusion.

Comparing (2) and (4), the intrinsic effective binary diffusivity ( $\mathcal{D}$, the subscript $i$ has been dropped to simplify the notation) for a given component can be related to the concentration-based effective binary diffusivity $(D)$ in one-dimensional case through the following:

$$
D=\mathcal{D}\left(1+\frac{\partial(\ln \gamma) / \partial x}{\partial(\ln C) / \partial x}\right)
$$

Equation (5) is equivalent to Darken's equation [Shewmon, 1963, p. 126]. The differential term in (5) is taken in a compositional gradient of major components and hence is unlikely to be zero even for trace components (unless $\gamma_{\text {is independent of not only } C}$ but also concentrations of other major components). Since Darken's equation relates a binary diffusivity $(D)$ to a selfdiffusivity, the intrinsic diffusivity defined in (4) is equivalent to the self-diffusivity in a binary system. When $\gamma$ is constant, $\mathcal{D}$ equals $D$. For uphill diffusion profiles during spinodal decomposition in a binary system, or for uphill diffusion profiles in multicomponent systems caused by variable $\gamma, D$ varies from positive to negative even if $\mathcal{D}$ is constant.

The activity coefficient $\gamma$ in (4) can be related to the concentration of the component at equilibrium or quasiequilibrium $\left(C_{e}\right)$ in a system using the two-liquid partition concept [Watson, 1982]. Figure 1a shows the case for two immiscible liquids or two liquids in which $\mathrm{SiO}_{2}$ and/or $\mathrm{Al}_{2} \mathrm{O}_{3}$ (which, to a large extent, determine the structure of silicate melts and hence the partitioning behavior of other elements) diffuse at a rate infinitely slow compared to other components. Other major or trace components partition in the two liquids so that their activities in the two liquids are the same and the partitioning can be characterized by equilibrium partition coefficients. Since the activity $(a=\mathcal{K})$ ) of a component is uniform at equilibrium (when the same standard state is used for the two liquids), the activity coefficient $\gamma_{e}$ (i.e., $\gamma$ at equilibrium) is inversely proportional to the equilibrium concentration. For convenience in later derivations, the concentration difference of the component between the two liquids at equilibrium is referred as $2 C_{f}$ (i.e., $C_{f}$ $=\left(C_{+, e}-C_{-, e}\right) / 2$, where $C_{+, e}$ and $C_{-, e}$ are the concentrations at the right- and left-hand halves at equilibrium). The term $C_{f}$ can be related to the two-liquid partition coefficient $K$ by the following form:

$$
K=C_{+, d} d C_{-, e}=\left(C_{0}+C_{f}\right) /\left(C_{0}-C_{f}\right),
$$

where $C_{0}=\left(C_{+, e}+C_{-, e}\right) / 2$. 

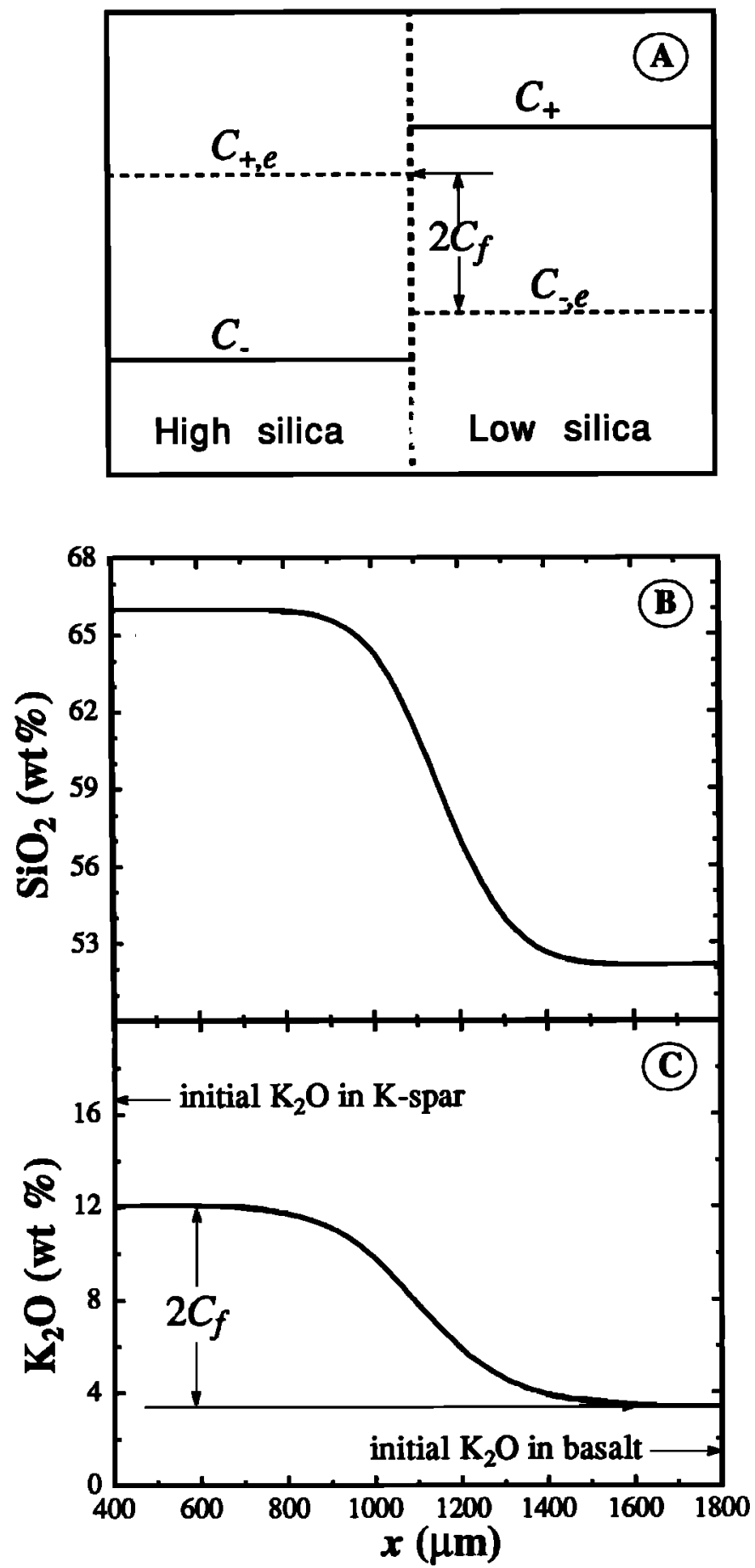

Fig. 1. The concept of $C_{e}$. (a) The two liquids are immiscible. Initial concentration distribution is $C_{+}$for $x>0$ and $C_{-}$for $x<0$. At equilibrium the concentrations are $C_{+, e}$ for $x>0$ and $C_{-, e}$ for $x<0$. Note that at equilibrium the profile is reversed and is a step function. The difference in the equilibrium concentration between the two liquids is termed $2 C_{f}$. (b) $\mathrm{SiO}_{2}$ profile in a compositional continuum. (c) $C_{e}$ in a compositional continuum. $\mathrm{K}_{2} \mathrm{O}$ profile in this case is close to a quasiequilibrium distribution because $\mathrm{K}_{2} \mathrm{O}$ concentrations at the ends are flat and different from the initial concentrations. Note that the $\mathrm{K}_{2} \mathrm{O}$ profile mimics $\mathrm{SiO}_{2}$ profile. Figures $1 \mathrm{~b}$ and $1 \mathrm{c}$ are adapted from Watson [1982].

Now, imagine that the number of liquids is infinite, so that the liquids comprise a compositional continuum in which $\mathrm{SiO}_{2}$ and/or $\mathrm{Al}_{2} \mathrm{O}_{3}$ profiles are smooth (Figure $1 \mathrm{~b}$ ). If the $\mathrm{SiO}_{2}$ and/or $\mathrm{Al}_{2} \mathrm{O}_{3}$ profiles were fixed (i.e., time-independent), then other components would "partition" along the continuum of miscible liquids such that $a$ is constant. In this hypothetical state, each component would reach a quasi-equilibrium distribution among the continuum of liquids which is characterized by a constant $a_{e}$ $\left(=\gamma_{e} C_{e}\right)$. (This quasi-equilibrium distribution of a component in a continuum of liquids is not identical to the local equilibrium concept which means equilibrium of a reaction in a very small volume.) Hence $\gamma_{e}$ is inversely proportional to $C_{e}$. The concentration difference for the component between the two endmember liquids at quasi-equilibrium is again referred as $2 C_{f}$. A hypothetical two-liquid partition coefficient $K$ between the two end-member liquids ("hypothetical" because the two liquids are not immiscible) can be defined by (6) in which $C_{+, e}$ and $C_{-, e}$ are replaced by $C_{+\infty, e}$, and $C_{-\infty, e}$ (o with respect to $\mathrm{SiO}_{2}$ diffusion). Such a quasi-equilibrium partitioning may be approximately reached for $\mathrm{K}_{2} \mathrm{O}$ in feldspar-basalt diffusion couple experiments (Figure 1c, adapted from Watson [1982]) and for Sr in the 12hour experiment of Lesher [1990]. In other experiments or for other components [e.g., Watson, 1982; Zhang et al., 1989; Lesher, 1990], quasi-equilibrium partitioning is clearly not achieved. Whether or not such quasi-equilibrium distribution is reachable depends on whether or not the intrinsic diffusivity for the component is much greater than that for $\mathrm{SiO}_{2}$ and/or $\mathrm{Al}_{2} \mathrm{O}_{3}$. However, regardless of whether or not this state can be reached, it is always possible to use the fictive state of quasi-equilibrium distribution as a reference and compare the transient diffusion profile against it.

In general, $C_{e}$ (or $\gamma_{e}$ ) is a complicated function of the concentrations of all components, depending on the choice of components [e.g., Ghiorso et al., 1983; Ghiorso, 1987b]. At present, no solution model accurately describes the activitycomposition relationship of complicated silicate melts of at least six components. For example, activity profiles calculated from diffusion profiles of major components of Zhang et al. [1989] using the model of Ghiorso et al. [1983] show that for some components the activity profile is monotonic but the flux is against the activity gradient across the whole profile. (Note that this is not equivalent to uphill diffusion against an activity gradient because in the latter case there would be minimum and/or maximum points.) Such unreasonable results are due to uncertainties in the calculated activity profiles, i.e., in the mixing parameters in the model of Ghiorso et al. [1983]. Ghiorso et al. [1983] also showed that their model cannot reproduce two-liquid partition data for some components. Data on mixing properties of trace components are even more scarce. Hence an approximate method is employed to express the hypothetical equilibrium concentration of a major or trace component in a tractable form by assuming that $C_{e}$ is a linear or an exponential function of the concentration of $\mathrm{SiO}_{2}$ and/or $\mathrm{Al}_{2} \mathrm{O}_{3}$. Note that this is merely an approximation over a small compositional space and is not meant to be an accurate thermodynamic treatment. In fact, the simple linear or exponential function does not satisfy all thermodynamic constraints on activity coefficients [Ghiorso, 1987b]. However, this approximation can be derived from a regular solution model by ignoring all but one mixing term and then linearizing the expression. The linear or exponential approximation requires only one parameter for each additional component, while a regular solution model would require 28 parameters for an eight-component system. In the following discussions, both the linear and exponential expressions are given in the theoretical development. However, simulations and fittings reported in this work use the linear approximation for $C_{e}$ because fitting results of experimental data using the linear approximation are slightly better in most cases than those using the exponential approximation. (If desired, it is relatively straightforward to implement the exponential approximation in simulations and in 
fitting experimental data.) The validity of the linear approximation is supported by the observation that $\mathrm{Sr}$ and $\mathrm{Nd}$ concentrations are linear functions of $\mathrm{SiO}_{2}$ and/or $\mathrm{Al}_{2} \mathrm{O}_{3}$ when isotopic homogeneity is reached in the experiments of Lesher [1990], which is roughly equivalent to attaining a quasiequilibrium distribution for the concentration of the component (F.M. Richter, personal communication, 1991).

Since $\gamma$ is assumed to be a function of $\mathrm{SiO}_{2}$ and/or $\mathrm{Al}_{2} \mathrm{O}_{3}$ contents only (i.e., $\gamma$ is assumed to be independent of concentrations of minor components), the value of $\gamma$ is independent of whether or not the quasi-equilibrium state for the component is reached or reachable. That is, for a given transient profile of $\mathrm{SiO}_{2}$ and/or $\mathrm{Al}_{2} \mathrm{O}_{3}, \gamma=\gamma_{e} \propto 1 / C_{e}$. Hence (4) can be rewritten as

$$
J \approx-\mathcal{D} C_{e} \nabla\left(C / C_{e}\right) \text {. }
$$

All of the assumptions implicit in (4) are equally applicable to (7). It is emphasized that the validity of (7) is independent of the relative size of $\mathcal{D}$ and $D_{b}$, or whether or not the hypothetical quasi-equilibrium corresponds to any actual state. The hypothetical state is merely a convenient reference state based on which the activity is expressed.

For simplicity, it is further assumed that the diffusion of $\mathrm{SiO}_{2}$ and/or $\mathrm{Al}_{2} \mathrm{O}_{3}$ can be characterized by a concentrationindependent effective binary diffusivity $D_{b}$ [Cooper, 1968; Hofmann, 1980; Zhang et al., 1989]. The case for variable $D_{b}$ can be incorporated into the present model if a functional dependence of $D_{b}$ on bulk composition is assumed, such as an exponential function of $\mathrm{SiO}_{2}$ concentration [Watson and Baker, 1991]. However, if strong uphill diffusion occurs for $\mathrm{SiO}_{2}$ and/or $\mathrm{Al}_{2} \mathrm{O}_{3}$, this model should not be used. With constant $D_{b}$ the concentration profile for $\mathrm{SiO}_{2}$ and/or $\mathrm{Al}_{2} \mathrm{O}_{3}$ is an error function for an infinite diffusion couple with respect to $\mathrm{SiO}_{2}$ and/or $\mathrm{Al}_{2} \mathrm{O}_{3}$. Combining this assumption with the assumption that $C_{e}$ of the component in consideration is a linear function of the concentration of $\mathrm{SiO}_{2}$ and/or $\mathrm{Al}_{2} \mathrm{O}_{3}, \mathrm{C}_{e}$ is also an error function and can be written as

$$
C_{e}=\frac{C_{-}+C_{+}}{2}+C_{f} \text { erf } \frac{x}{2 \sqrt{D_{b} t}}
$$

where $C_{-}$and $C_{+}$are the initial concentrations at $x<0$ and $x>0$ for the diffusion couple. Justification for (8) can be found from the near error function shape of $\mathrm{K}_{2} \mathrm{O}$ profiles in experimental diffusion couples believed to be near quasi-equilibrium partitioning [Watson, 1982; adapted in Figure 1c]. Given a transient $\mathrm{SiO}_{2}$ and/or $\mathrm{Al}_{2} \mathrm{O}_{3}$ profile, $C_{e}$ profile can always be calculated using (8) if $C_{f}$ is known regardless of whether the quasi-equilibrium distribution is reached or reachable. Equation (8) is simple and conceptually clear in that there are only two parameters: $C_{f}$, which can be related to and estimated from twoliquid partition data, and $D_{b}$, which can be related to and estimated from the concentration-based effective binary diffusivity of $\mathrm{SiO}_{2}$ and/or $\mathrm{Al}_{2} \mathrm{O}_{3}$. Since the quasi-equilibrium distribution of different components may depend on different structural components (e.g., the activity of component 1 may be mostly dependent on $\mathrm{SiO}_{2}$ concentration, while component 2 may be mostly dependent on $\mathrm{Al}_{2} \mathrm{O}_{3}$ concentration), it is not necessary for $D_{b}$ of different components to be exactly the same.

Assuming $C_{e}$ to be an exponential function of the concentration of $\mathrm{SiO}_{2}$ and/or $\mathrm{Al}_{2} \mathrm{O}_{3}$, then

$$
C_{e}=\frac{C_{-}+C_{+}}{2} \mathrm{e}^{\operatorname{Berf} \frac{x}{2 \sqrt{D_{b} t}}},
$$

where the parameter $\beta$ is functionally similar to the parameter $C_{f}$ in (8) and can be related to the two-liquid partition coefficient. When $\beta \ll 1,(8)$ and $\left(8^{\prime}\right)$ are equivalent and $\beta\left(C_{-}+C_{+}\right) \approx 2 C_{f}$.

In summary, there are two major approximations in this model. One is to approximate (3) by an activity-based effective binary form (4), or its equivalent (7). The other major approximation is to relate the quasi-equilibrium partition concentration of a component to the concentration of $\mathrm{SiO}_{2}$ and/or $\mathrm{Al}_{2} \mathrm{O}_{3}$ by a linear or an exponential function. A simplification of constant $D_{b}$ is also employed to further simplify the approach. In an infinite diffusion couple, these approximations lead to (8) or (8'). The appropriate equation will be different for diffusion during mineral dissolution (discussed later) or crystal growth. If $D_{b}$ cannot be regarded as constant, further complication will occur but it can be readily incorporated into this model. This model is approximate and the range of applicability must be tested using experimental data, but it is more general than concentration-based effective binary treatments.

\section{Simulations OF DIFFUSIVE BEHAVIOR}

\section{Some General Remarks}

In the context of this model, diffusion of a component in a multicomponent system is described approximately by

$$
\frac{\partial C}{\partial t}=\frac{\partial}{\partial x}\left(\frac{\mathcal{D}}{\gamma} \frac{\partial(\gamma C)}{\partial x}\right),
$$

or its equivalent form

$$
\frac{\partial C}{\partial t}=\frac{\partial}{\partial x}\left(\mathcal{D C} C_{e} \frac{\partial\left(C / C_{e}\right)}{\partial x}\right)
$$

Unlike in flux equations where the unit of $C$ is mass/volume or mole/volume, the unit of $C$ in (9) may also be weight percent for constant total mass density $(\rho)$ since $\rho$ can be factored out. For constant total molar density, the unit of $C$ can be mole percent. In general, (9) is nonlinear and must be solved numerically unless $\boldsymbol{\gamma}$ (or $\left.C_{e}\right)$ is constant. If both concentration and activity profiles can be determined, (9) can be integrated for infinite diffusion couples in a manner similar to a Boltzmann-Matano analysis [Shewmon, 1963] so as to calculate $\mathcal{D}$. Defining $\eta=x / \sqrt{4 t}$, (9a) can be transformed into

$$
-2 \eta \frac{d C}{d \eta}=\frac{d}{d \eta}\left(\frac{\mathcal{D}}{\gamma} \frac{d a}{d \eta}\right)
$$

which upon integration from $\eta_{0}$ to $\infty$ gives

$$
2 \int_{C\left(\eta_{0}\right)}^{C(\infty)} \eta d C=\frac{\mathcal{D}}{\gamma} \frac{\mathrm{d} a}{\mathrm{~d} \eta} \mathrm{l}_{\eta_{0}},
$$

because $\left.(\mathrm{d} a / \mathrm{d} \eta)\right|_{\eta=\infty}=0$ for an infinite diffusion couple. Therefore $\mathcal{D}$ can be calculated from the activity gradient on the right-hand side and concentration integration on the left-hand side of (11). A negative $\mathcal{D}$ calculated from (11) indicates intrinsic uphill diffusion, which is caused by cross-term diffusivities in (3) and cannot be treated by this model.

The activity of a component does not follow a simple diffusion equation, i.e.,

$$
\frac{\partial a}{\partial t} \neq \frac{\partial}{\partial x}\left(\frac{\mathcal{D}}{\gamma} \frac{\partial a}{\partial x}\right)
$$

but rather satisfies

$$
\frac{\partial a}{\partial t}=\gamma \frac{\partial C}{\partial t}+C \frac{\partial \gamma}{\partial t}=\gamma \frac{\partial}{\partial x}\left(\frac{\mathcal{D}}{\gamma} \frac{\partial a}{\partial x}\right)+C \frac{\partial \gamma}{\partial t} .
$$


Therefore activity profiles can show minimum and maximum points even though it is assumed that mass always diffuses down activity gradients. This arises because of the complicated relationship between $a$ and $C$ and because $C$ is on the left-hand side of (9), while $a$ (i.e., $\gamma_{C}$ or $C / C_{e}$ ) is on the right-hand side. Consider, for example, the case in which activity is initially constant across the whole profile. (In this case, it is necessary to consider cross terms, however small they may be, and hence the discussion is purely academic and may be unrealistic.) Hence in (13), $\partial a /\left.\partial x\right|_{t=0}=0$, but $\partial \gamma / \partial t \neq 0$ as the concentration of $\mathrm{SiO}_{2}$ and/or $\mathrm{Al}_{2} \mathrm{O}_{3}$ change. Changes in $a$ would occur with time according to (13), resulting in extreme points in the activity profile. Even in the presence of the extreme points in the activity profile, the diffusive flux is still always down its activity gradient in the context of this model. Detailed examples will be given in the following sections.

The model presented above can be used to model diffusion during spinodal decomposition, diffusion across two immiscible liquid phases, mixing and unmixing of two liquids, diffusion in a

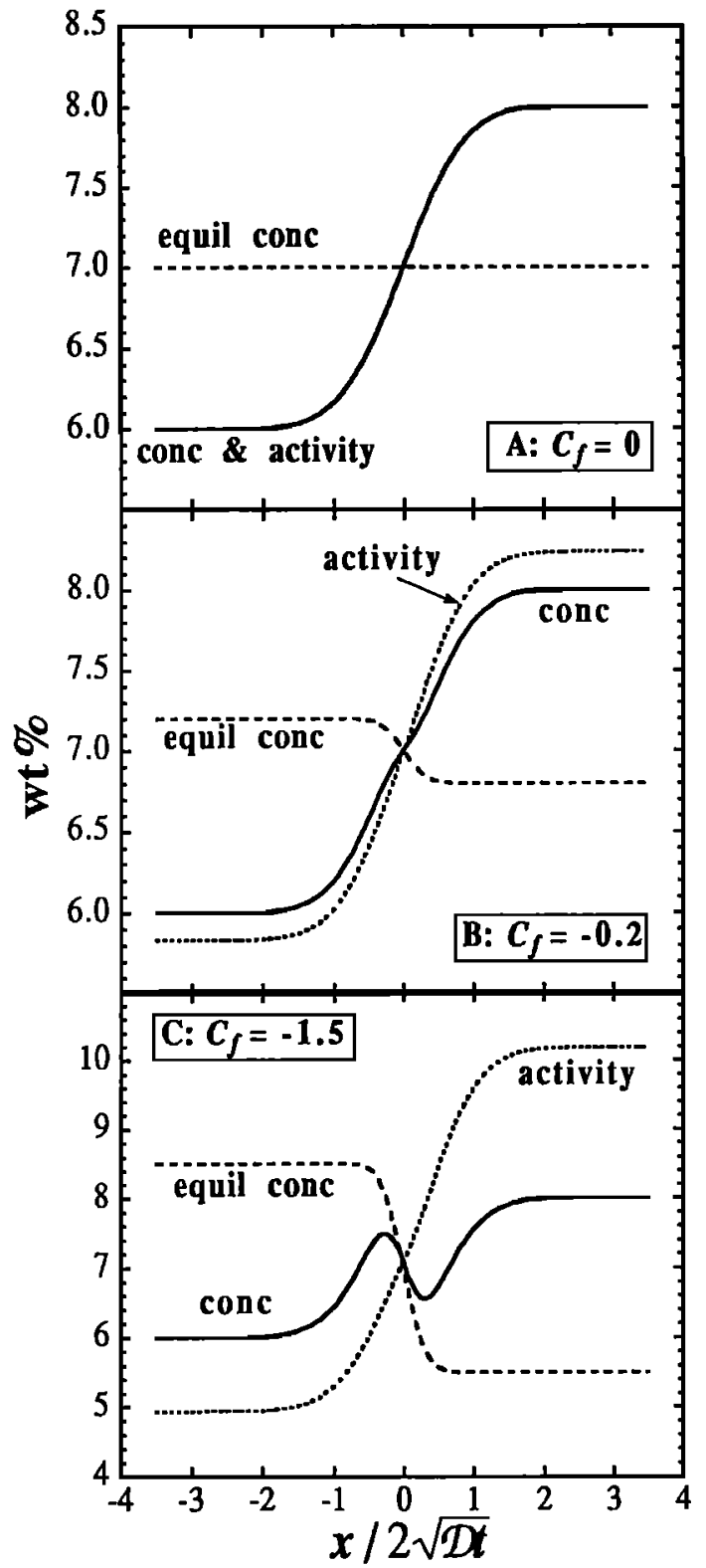

diffusion couple, isotopic and chemical diffusion, diffusion during crystal dissolution and growth, etc. In the first three cases, treatment by this model is simpler than previous treatments [cf. Kirkaldy and Young, 1987] because it is not necessary to trace phase boundaries if surface effects are not important. In this paper, three simulation problems are selected to illustrate the model: (1) chemical diffusion in a diffusion couple, (2) isotopic and chemical diffusion, and (3) diffusion during crystal dissolution and growth.

\section{Chemical Diffusion Across a Diffusion Couple}

Diffusion across an infinite diffusion couple in a multicomponent system is modeled by (9b) with $C_{e}$ given by (8). Given initial conditions, the problem can be solved numerically. An explicit finite difference technique is used to solve the diffusion equation [Press et al., 1989]. A simulation example is shown in Figure 2 for $D / D_{b}=8, C_{-}=6 \%$ and $C_{+}=8 \%$. $C_{f}$ is varied to show how concentration profiles depend on the partition

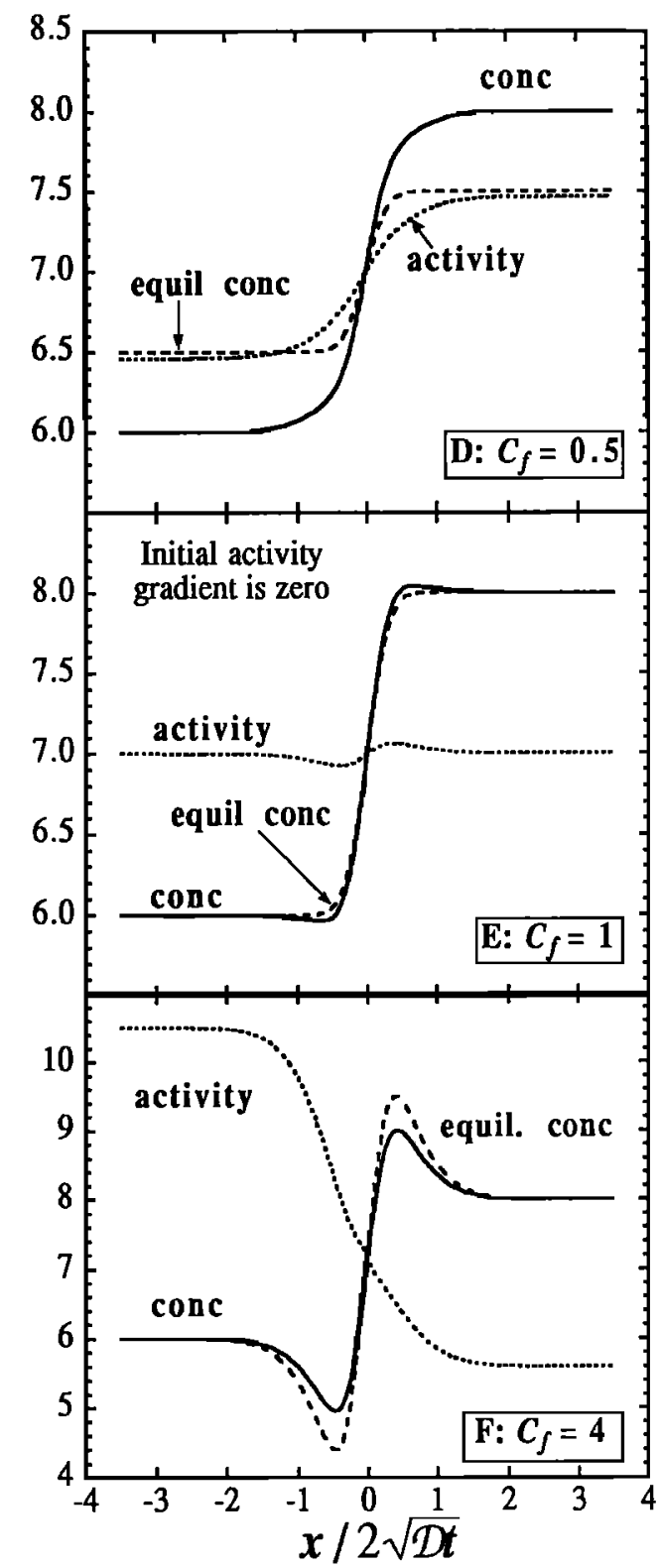

Fig. 2. Calculated concentration (solid curves), activity (dotted curves) and the quasi-equilibrium concentration (dashed curves) profiles for a diffusion couple for $C_{-}=6 \%$ and $C_{+}=8 \%$ with $D / D_{b}=8$. At $x=0$ (interface), $a$ is defined to equal $C$. (a) $C_{f}=0$, i.e., $a \propto C$; (b) $C_{f}=-0.2$; (c) $C_{f}=-1.5$; (d) $C_{f}=0.5$; (e) $C_{f}=1$, i.e., initially, $\Delta a=0$; (f) $C_{f}=4$. 
behavior. For the special case $C_{f}=0$, i.e., when $a$ is proportional to $C$, there is an analytical solution which is an error function. The calculated profile (Figures $2 a$ and $3 a$ ) based on numerical solution of (9b) is identical to the analytical solution to within $3 \times 10^{-5}$ ( $2 \sigma$ relative error), a rough estimate of the numerical error

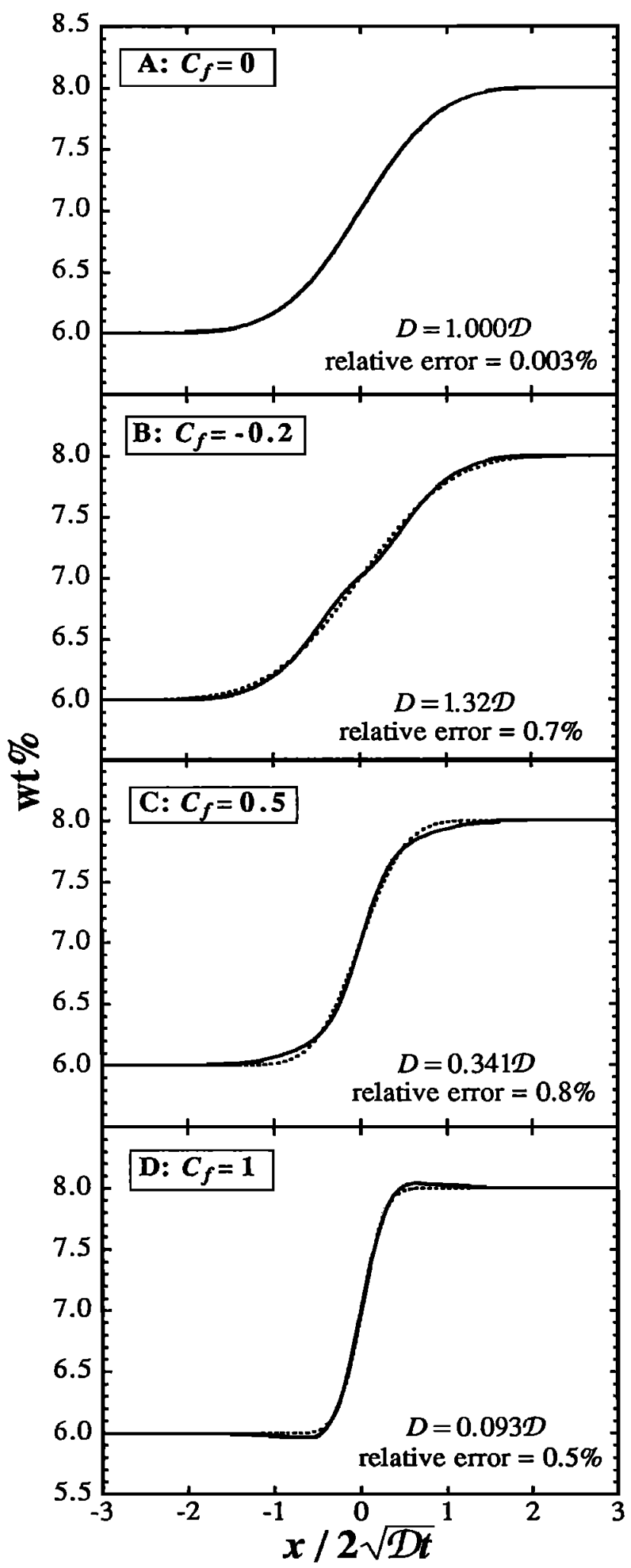

Fig. 3. Error function fits (dashed curves) to calculated diffusion couple profiles (solid curves) for (a) $C_{f}=0$, (b) -0.2 , (c) 0.5 , and (d) 1 . Initially, $C_{-}=6 \%$ and $C_{+}=8 \%$. $D_{b} / \mathcal{D}=0.125$. The best fit $D$ is given as a fraction of $\mathcal{D}$. Relative errors $(2 \sigma)$ in fittings are also shown. in the calculated solution for the diffusion equation for the space and time steps chosen for the numerical calculations. As $C_{f}$ is decreased from 0 to negative (Figures $2 b$ and $2 c$ ), the activity gradient has the same sign as the concentration gradient but is greater in magnitude; that is, the hypothetical $C_{e}$ gradient is opposite to the concentration gradient. Hence mass diffuses from the high concentration side to the low concentration side at a higher rate than if $C_{f}=0$, resulting in either a pair of bumps in the concentration profile when $\left|C_{f}\right|$ is very small (e.g., $\leq 0.2$, see Figure $2 b$ ) or a maximum on the lower concentration side and a minimum on the higher concentration side (Figure 2c). This effect increases as $C_{f}$ becomes more negative.

On the other hand, as $C_{f}$ increases from 0 to $1 \%$, the sign of the activity and concentration gradients is the same but the activity gradient becomes progressively smaller. In this $C_{f}$ range, the deviation of the concentration profile from an error function is small (Figures 2d and 2e). In other words, it would be difficult to distinguish such an experimental profile from an error function profile or to constrain both $\mathcal{D}$ and $C_{f}$ from it, especially when analytical errors and the variability of the diffusivity are considered (see Figure 3). As $C_{f}$ approaches 1 , the profile becomes shorter and has a length characterized by $D_{b}$ instead of $\mathcal{D}$ (Figure 2e). When $C_{f}=1$, the initial activity gradient is zero. However, as the concentrations of $\mathrm{SiO}_{2}$ and $\mathrm{Al}_{2} \mathrm{O}_{3}$ evolve, the activity gradient becomes nonzero and diffusion occurs. As a result, a maximum and a minimum develop over the activity profile (Figure 2e). The diffusive flux is always down the activity gradient. For example, at the maximum, the flux is from the maximum to both spatial directions. The concentration profile also displays a small minimum and a small maximum; i.e., there is uphill diffusion. Activity and concentration extremes do not correspond to each other. Rather, activity extremes (where $\mathrm{d} a / \mathrm{d} x=0$ ) correspond to points in the concentration profile where $\int x d C$ from that point to $C_{\infty}$ is 0 , a necessary condition to satisfy (11) with a constant $\mathcal{D}$. For $C_{f}>1 \%$, the sign of the activity gradient is opposite to that of the concentration gradient, and uphill diffusion occurs (Figure 2f), the intensity of which increases with $C_{f}$. The activity profile is almost always monotonic and resembles an error function unless initial $\Delta a=a_{+}$ $-a_{-} \approx 0$ (i.e., $C_{f} \approx 1$ ). The length of the activity profile roughly reflects the intrinsic $\mathcal{D}$. If one were to fit an error function through the activity profiles, the apparent $D$ would agree with the intrinsic $\mathcal{D}$ within a factor of two.

The above discussion can be viewed in another way. The activity of a given component (such as $\mathrm{MgO}$ ) depends on the concentrations of $\mathrm{SiO}_{2}$ and $\mathrm{Al}_{2} \mathrm{O}_{3}$. Let $\mathrm{SiO}_{2}$ and $\mathrm{Al}_{2} \mathrm{O}_{3}$ concentrations for the two halves of the diffusion couple be fixed. Hence the $C_{f}$ can be regarded as a constant. Let initial $\Delta C_{\mathrm{MgO}}$ vary from one experiment to another (the variation can be compensated by the variation of, e.g., $\mathrm{CaO}$ ). Therefore, as the $\Delta C_{\mathrm{MgO}}$ changes, $\Delta a_{\mathrm{Mg}}$ changes such that $\Delta a$ and $\Delta C$ sometimes have the same sign and some times have the opposite sign. There will be uphill diffusion in some cases, and monotonic profiles in other cases. Concentration-based effective binary diffusivities extracted from different monotonic profiles will be different. This provides one of the experimental tests of this model.

Table 1 summarizes the diffusive behavior from simulation results. When either $\Delta a$ or $\Delta C_{e}$ is initially in the opposite direction to $\Delta C$, there is uphill diffusion unless $\Delta C_{e}$ is very small. In the region in the $\Delta a$ versus $\Delta C$ space where $\Delta a$ and $\Delta C_{e}$ are both initially in the same direction as $\Delta C$, the diffusion profile is affected by variable $\gamma$ but the concentration profile remains monotonic. In this region, the effect of variable $\gamma$ is difficult to detect based purely on the shapes of the concentration profiles. If an error function was fit to obtain a $D$ from such a profile, the fit would be good within error. Therefore it is difficult to 
TABLE 1. Summary of Simulation Results

\begin{tabular}{ccc}
\hline$C_{f}$ range & Roughly equivalent to: & Uphill diffusion? \\
\hline \multicolumn{3}{c}{ Diffusion Couple, $C_{-\infty}=6, C_{+\infty}=8, \mathcal{D} / D_{b}=8$} \\
$C_{f}>0.9 \quad \Delta a$ opposite to $\Delta C$ & Yes \\
$-0.2 \leq C_{f} \leq 0.9$ & $\Delta a, \Delta C_{e}$ and $\Delta C$ have same sign & No \\
$C_{f}<-0.2$ & $\Delta C_{e}$ opposite to $\Delta C$ & Yes \\
$C$ Crystal Dissolution, $C_{s}=0, C_{\infty}=8, A=0.3, \mathcal{D} D_{b}=8$ \\
$C_{f}>0.5$ & Yes \\
$-2 \leq C_{f} \leq 0.5$ & No \\
$C_{f}<-2$ & Yes \\
$C r y s t a l$ & Dissolution, $C_{s}=50, C_{\infty}=4, A=0.3, \mathcal{D I} D_{b}=8$ \\
$C_{f}<-0.5$ & Yes \\
$-0.5 \leq C_{f} \leq 15$ & No \\
$C_{f}>15$ & Yes
\end{tabular}

$\Delta a, \Delta C_{e}$ and $\Delta C$ refer to initial conditions for a diffusion couple. The numerical factors apply only to the chosen simulation conditions.

distinguish these diffusion profiles from those with truly constant $\gamma$.

Though the monotonic concentration profiles generally cannot be distinguished with any confidence from an error function profile when $0.1 \Delta C \leq \Delta a \leq 1.1 \Delta C$ (recall that $\left.a\right|_{x=0}=\left.C\right|_{x=0}$ ), $D$ for the component obtained by fitting an error function to the profile changes from greater than $\mathcal{D}$ (when $\Delta C_{e}$ and $\Delta C$ are initially in opposite directions but $\Delta C_{e}$ is very small) to smaller than $D_{b}$ (when $\Delta a \approx 0$ ). Figure 3 shows how the best fit $D$ changes with $C_{f}$ (which determines the relationship between $\Delta a$ and $\Delta C$ ). When $C_{f}=0$ (i.e., when $a$ is proportional to $C$ ), the calculated profile (Figure 3a) is the same as the analytical solution such that $D=\mathcal{D}$. When $C_{f}$ changes from 0 to $-0.2, D / \mathcal{D}$ changes from 1 to 1.32 (Figure 3b). When $C_{f}$ changes from 0 to $1, D / \mathcal{D}$ changes from 1 to 0.093 (Figures 3c and 3d) (recall that in the calculation, $D_{b} / \mathcal{D}=1 / 8=0.125$ ). All error function fits of calculated profiles shown in Figure 3 are better than the precision in electron microprobe analysis $(\sim 1 \%$ relative) that are generally used to measure major element profiles and much better than the $\sim 5 \%$ relative precision that can be expected for routine trace element analysis by ion microprobe techniques. Therefore fitting a monotonic experimental concentration profile gives a $D$ that depends on the size of the initial activity gradient and lies somewhere between $\sim 1.3 \mathcal{D}$ and $\sim D_{b} / 1.3(\sim 0.1 \mathcal{D})$ in this case (i.e., $\left.D_{b} / 1.3 \leq D \leq 1.3 \mathcal{D}\right)$.

The above results apply only to the case when $\mathcal{D}>D_{b}$. If $D_{b}>\mathcal{D}$, then some other complications occur (e.g., length of concentration profiles reflects $\mathcal{D}$ better than length of activity profiles). The results are not discussed here because such cases are not common for silicate melts.

\section{Comparison of Isotopic Ratio Profiles and Chemical Concentration Profiles}

The development of chemical concentration and isotopic ratio profiles during diffusion can also be modeled using this model. For an infinite diffusion couple, the concentration of each isotope of an element satisfies the diffusion equation (9), and all the isotopes have essentially the same activity coefficient which relates $C_{e}$ of different isotopes. The concentration profile for each isotope can be calculated directly from (9b) and thereby the isotopic ratio profile can be calculated by taking the ratio of two isotopes. Examples of calculated isotopic ratio and chemical concentration profiles are shown in Figure 4. In this simulation, initial conditions similar to those reported by Lesher [1990] are used. The simulation results can be summarized as follows.

1. To a first-order approximation, the length of the isotopic profile reflects the intrinsic diffusivity. If the profile is fit by an

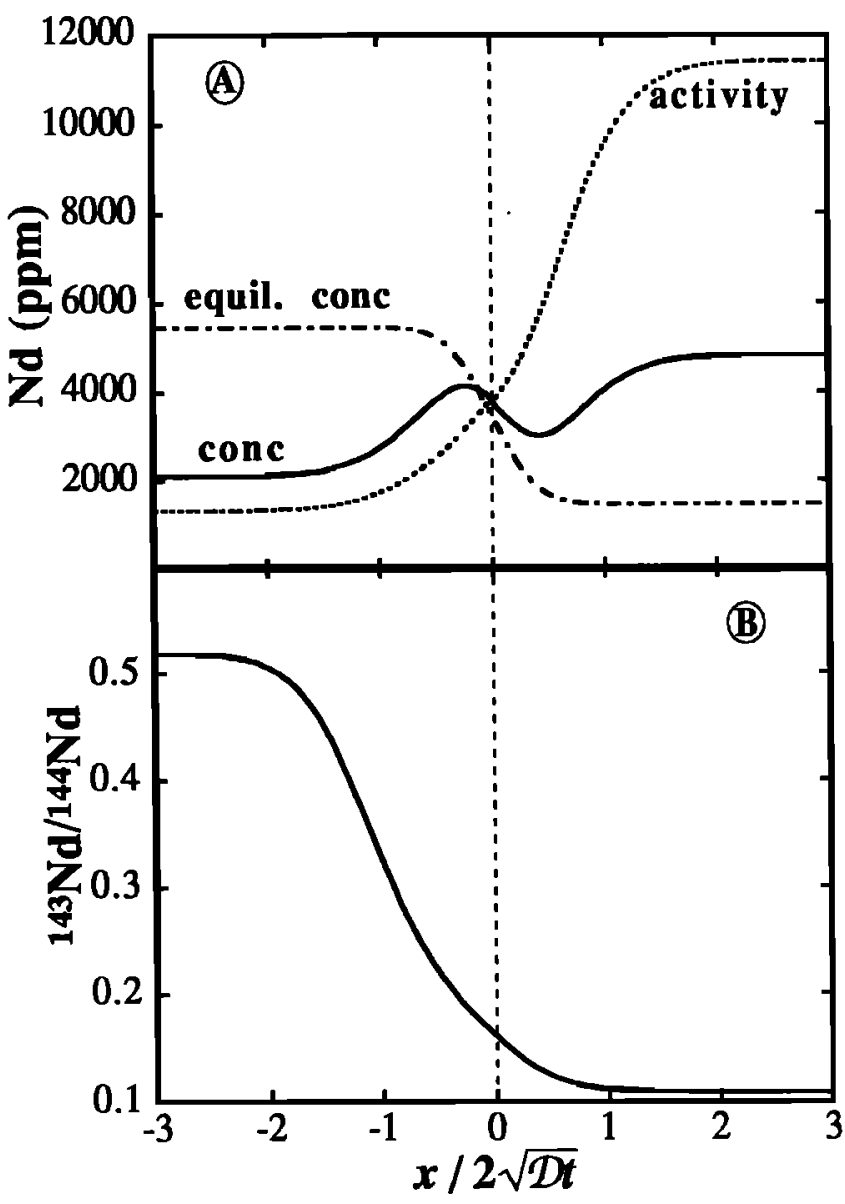

Fig. 4. Calculated concentration and isotopic profiles for a diffusion couple for $C_{-}=2110 \mathrm{ppm}$ and $C_{+}=4825 \mathrm{ppm},\left({ }^{\left.143 \mathrm{Nd} /{ }^{144} \mathrm{Nd}\right)_{-}=0.5147}\right.$ and $\left({ }^{143} \mathrm{Nd} / 144 \mathrm{Nd}\right)_{+}=0.1075$, with $\mathcal{D} / D_{b}=5$. These conditions are chosen so as to roughly simulate the experimental results of Lesher [1990]. Vertical dashed line is the interface between the two halves. When compared to Lesher's results, the shape of the simulated profile is more symmetric than experimental profiles. The misfit is likely due to compositional dependence of $D_{b}$ and $\mathcal{D}$. See the work by Lesher [1991] and Richter and Stolper [1991] for more discussion.

error function by artificially setting the interface of the profile to the midpoint of the profile, the resulting diffusivity often agrees with actual intrinsic diffusivity to within $\pm 20 \%$. In fact, simulation results suggest that an even better approximation of the intrinsic diffusivity can be obtained by fitting the profile of a ratio of an isotope to total elemental concentration (e.g., ${ }^{143} \mathrm{Nd} / \Sigma \mathrm{Nd}$ ). In contrast, the length of the chemical concentration profile usually reflects a $D$ that can be greater than $\mathcal{D}$ or smaller than $D_{b}$ even if uphill diffusion does not show up, as discussed earlier.

2. The isotopic ratio profile is always monotonic; "uphill diffusion" of isotopic ratios is never generated in simulations, consistent with experimental results [Lesher, 1990; van der Laan et al., 1990]. Therefore the nonmonotonic ${ }^{87} \mathrm{Sr} /{ }^{86} \mathrm{Sr}$ and ${ }^{143} \mathrm{Nd} /{ }^{144} \mathrm{Nd}$ profiles observed by Davidson et al. [1990] either are not controlled by purely diffusive processes or require more complicated diffusion models. In contrast, chemical concentration profiles, or the concentration profile of an isotope, can show uphill diffusion under appropriate conditions.

3. The isotopic ratio profile may not be centered at the interface because concentrations of the element on the two sides are not identical. This is consistent with the results of Lesher [1990]. In contrast, the chemical concentration profile is roughly centered at the Matano interface. 
4. Isotopic ratio gradients may become homogenized much more rapidly than chemical concentration gradients [Hofmann and Hart, 1978; Baker, 1989, Lesher, 1990; van der Laan et al., 1990]. This arises because the length of the isotopic profile roughly reflects the intrinsic diffusivity $\mathcal{D}$, whereas the length of the chemical concentration profile corresponds to an apparent $D$ that can be slightly greater than $\mathcal{D}$ or smaller than $D_{b}$ and can be vastly smaller than the intrinsic diffusivity if $D_{b} \ll \mathcal{D}$.

Although this model can explain many features of isotopic and chemical diffusion experiments, the simulation shown in Figure 4 does not exactly match the experimental profiles of Lesher [1990], likely due to the nonapplicability of constant $\mathcal{D}$ and $D_{b}$ in this case [Lesher, 1991; F.M. Richter, personal communication, 1991] since the $\mathrm{SiO}_{2}$ content changes from $49 \%$ to $73 \%$ across the profile of the experiment. When the variation in $\mathrm{SiO}_{2}+\mathrm{Al}_{2} \mathrm{O}_{3}$ content in the profile is small, this model can fit both the isotopic profile and the concentration profile quantitatively (S. van der Laan et al., submitted manuscript).

\section{Chemical Diffusion During Diffusive Crystal Dissolution}

The diffusion equation for one dimensional diffusion in the melt during diffusive crystal dissolution in an interface-fixed reference frame is [Zhang et al., 1989]

$$
\frac{\partial C}{\partial t}=\frac{\partial}{\partial x}\left(\mathcal{D} C_{e} \frac{\partial\left(C / C_{e}\right)}{\partial x}\right)-V \frac{\partial C}{\partial x},
$$

where $V$ is the melt growth rate given by

$$
V=A / \sqrt{t},
$$

where $A$ is positive and constant (when $p$ is constant) characterizing the dissolution rate. Mass density $\rho$ is assumed to be constant in the calculations so that $A$ is constant and that unit of $C$ can be weight percent. This appears reasonable for most experiments conducted by Zhang et al. [1989]. For example, using partial molar volumes from Lange and Carmichael [1987], total density variation is $\sim 4 \%$ relative across the concentration profiles produced by San Carlos olivine dissolution into andesitic melt at $1285^{\circ} \mathrm{C}$ and $0.55 \mathrm{GPa}$ (run 212 of Zhang et al. [1989]; all run numbers hereafter indicate experiments in Zhang et al. [1989] unless otherwise specified, except for run 246 which is from Zhang et al., unpublished data, 1989). Because it is difficult to treat density variations across a diffusion profile, the present analysis should not be used to describe systems with large density variations ( $>10 \%$ relative variations).

Consider an initial condition

$$
\left.C\right|_{t=0}= \begin{cases}C_{\infty} & x>0 \text {, i.e., in the melt, } \\ C_{s} & x<0, \text { i.e., in the crystal, }\end{cases}
$$

with a boundary condition at $x=0$ :

$$
\mathcal{D C} \frac{\partial\left(C / C_{e}\right)}{\partial x}+V\left(C_{s}-C_{0}\right)=0
$$

where $C_{e}$ is given by

$$
C_{e}=C_{\infty}+C_{f} \operatorname{erfc} \frac{x / \sqrt{4 t}-A}{\sqrt{D_{b}}},
$$

which is written by analogy to the analytical solution for diffusive crystal dissolution with constant $D_{b}$ (equation (13) of Zhang et al. [1989]) assuming $C_{e}$ is a linear function of the concentration of $\mathrm{SiO}_{2}$ and/or $\mathrm{Al}_{2} \mathrm{O}_{3} . C_{f}$ is the concentration difference between the initial melt and the melt at the original interface at the hypothetical quasi-equilibrium state. Since in this case the medium is semi-infinite, the difference is defined as $C_{f}$, instead of
$2 C_{f}$ for the case of infinite medium. If $C_{e}$ is assumed to be an exponential function of the concentration of $\mathrm{SiO}_{2}$ and/or $\mathrm{Al}_{2} \mathrm{O}_{3}$, then the expression for $C_{e}$ is

$$
C_{e}=C_{\infty} \mathrm{e}^{\beta e f \mathrm{x} \frac{x / \sqrt{4 t}-A}{\sqrt{D_{b}}}} .
$$

Again, when $\beta \ll 1,(18)$ and (18') are equivalent and $\beta C_{\infty} \approx C_{f}$.

In order to extract $\mathcal{D}$ values from measured concentration and activity profiles, equations (14) to (16) can be transformed by Boltzmann-Matano analysis (with $\eta=x / \sqrt{4 t}$ ) into

$$
2 \int_{C\left(\eta_{0}\right)}^{C(\infty)}(\eta-A) d C=\left.D C_{e} \frac{\mathrm{d}\left(C / C_{e}\right)}{\mathrm{d} \eta}\right|_{\eta_{0}} \text {. }
$$

The $\mathcal{D}$ values as a function of composition (or distance) can be calculated from the integral on the left-hand side and the derivative on the right-hand side of (19) if $C_{e}$ is known. If a negative $\mathcal{D}$ is obtained, it indicates intrinsic uphill diffusion, which cannot be treated by this model.

Equations (14) to (18) can be solved numerically given $C_{s}, C_{\infty}$, $C_{f}, A, D_{b}$, and $\mathcal{D}$ (which are all assumed to be constant) using an explicit finite difference method [Press et al., 1989]. Two simulation results are given in Figures 5 and 6 . The concentrations of the component in the crystal and in the initial melt are chosen to be 0 and $8 \%$ in the first example (Figure 5) and $50 \%$ and $4 \%$ in the second example (Figure 6 ). The activity and $C_{e}$ of a component at $x=\infty$ (i.e., far away from the interface) are defined to be the same as the concentration of the component. The concentration profile in general mimics the $C_{e}$ profile because the component tries to reach the "equilibrium" distribution. The results are summarized in Table 1.

On the basis of the simulations, for components which are initially enriched in the melt (Figure 5), it is more likely for components with $C_{f}>0$ (such as nonalkaline components during olivine dissolution into andesitic melts) to display uphill diffusion. The $\mathcal{D}$ and $C_{f}$ parameters for these components are well constrained because relatively small changes in $C_{f}$ have a substantial effect on the resulting concentration profile. On the other hand, it is more difficult for components with $C_{f}<0$ (such as alkaline components during olivine dissolution into andesitic melts) to display uphill diffusion. The mere existence of a maximum point for an alkaline component shows that $\left|C_{f}\right|$ is relatively large, but its value is poorly constrained because a relatively large change in $C_{f}$ causes only small changes in the concentration profiles (Figure 5). It can also be shown that $\mathcal{D}$ values are poorly constrained from these profiles.

On the basis of Figure 6, for a principal equilibriumdetermining component [Zhang et al., 1989], such as MgO during olivine dissolution, an unusually large $C_{f}(\geq 20$ for the parameters chosen in Figure 6) is required for uphill diffusion if $C_{f}$ is positive (i.e., if the component partitions preferentially into the interface melt instead of the melt far away). If, however, $C_{f}$ is negative (i.e., if the component partitions preferentially into the melt far away from the interface), uphill diffusion can readily develop (e.g., Figures 6c and 6d). No uphill diffusion was observed for principal equilibrium-determining components by Zhang et al. [1989] because they all partition preferentially into the interface melt.

Summarizing the results of numerical simulation for diffusion in both infinite (diffusion couple) and semi-infinite (crystal dissolution) media, uphill diffusion occurs when either $\nabla a$ or $\nabla C_{e}$ is in the opposite direction of the nominally expected direction of $\nabla C$ unless $\nabla C_{e}$ is very small compared to $\nabla C$. It is possible to resolve both $\mathcal{D}$ and $C_{f}$ from such uphill concentration profiles even though in some cases $\mathcal{D}$ and $C_{f}$ are not well constrained. 

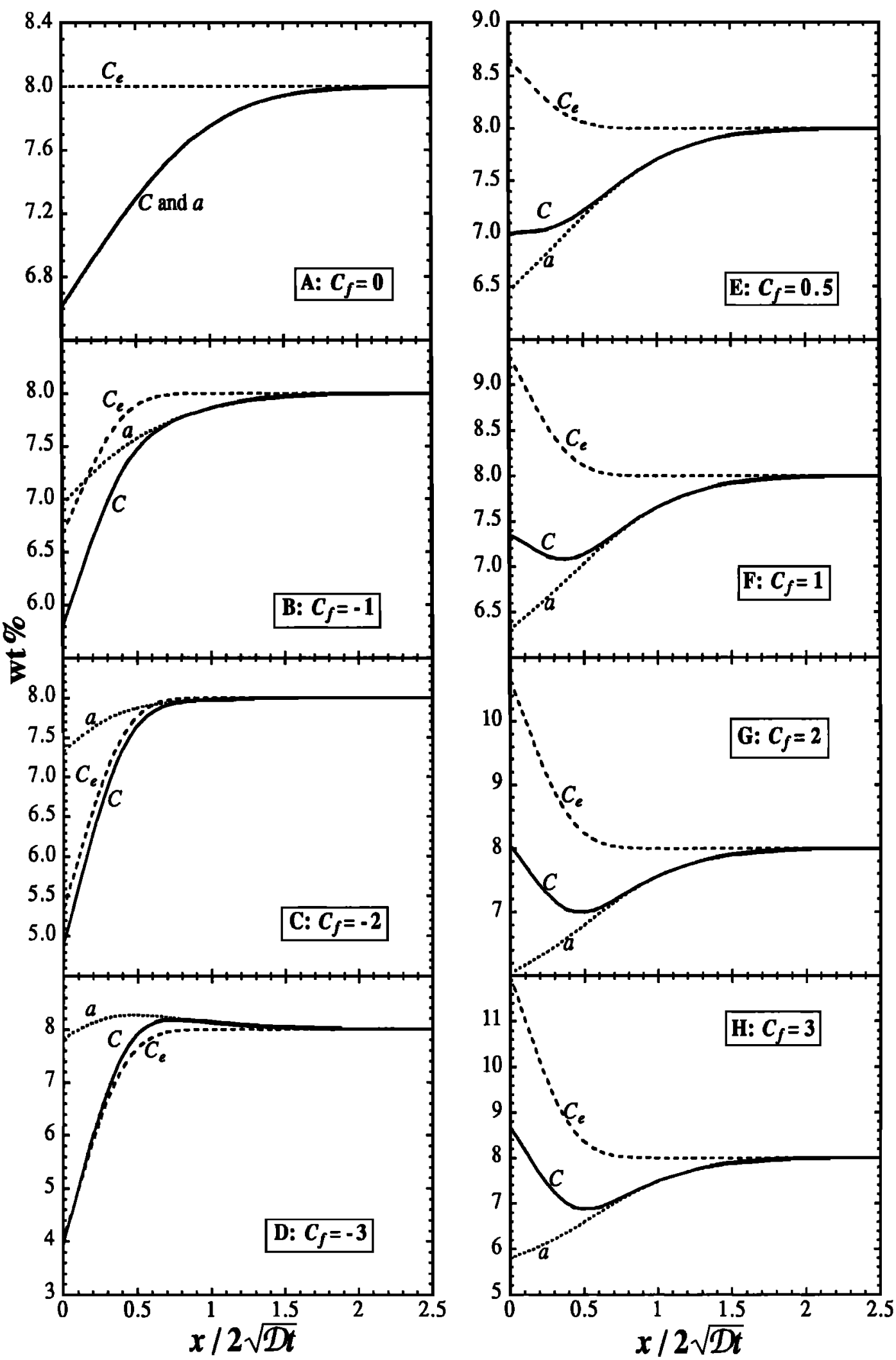

Fig. 5. Calculated $C$ (solid curves), $a$ (dotted curves) and $C_{e}$ (dashed curves) profiles for crystal dissolution for $C_{\infty}=8 \%$ and $C_{s}=0 \%$ with $\mathcal{D} / D_{b}=8$ and $A=0.3 \mu \mathrm{m} \mathrm{s} \mathrm{s}^{-1 / 2}$. At $x=\infty, a$ is defined to equal $C$.

When both $\nabla a$ and $\nabla C_{e}$ are in the same direction as the expected $\nabla C$, the concentration profiles are monotonic unless $\nabla a$ approaches zero. Such a profile would be fit well by a concentration-based effective binary treatment and $D$ obtained in this way would change from slightly greater than $\mathcal{D}$ to slightly smaller than $D_{b}$ as $\nabla a$ changes from slightly greater than $\nabla C$ to 0 . It is almost impossible to resolve both $\mathcal{D}$ and $C_{f}$ from such profiles unless one of the two can be determined independently. 

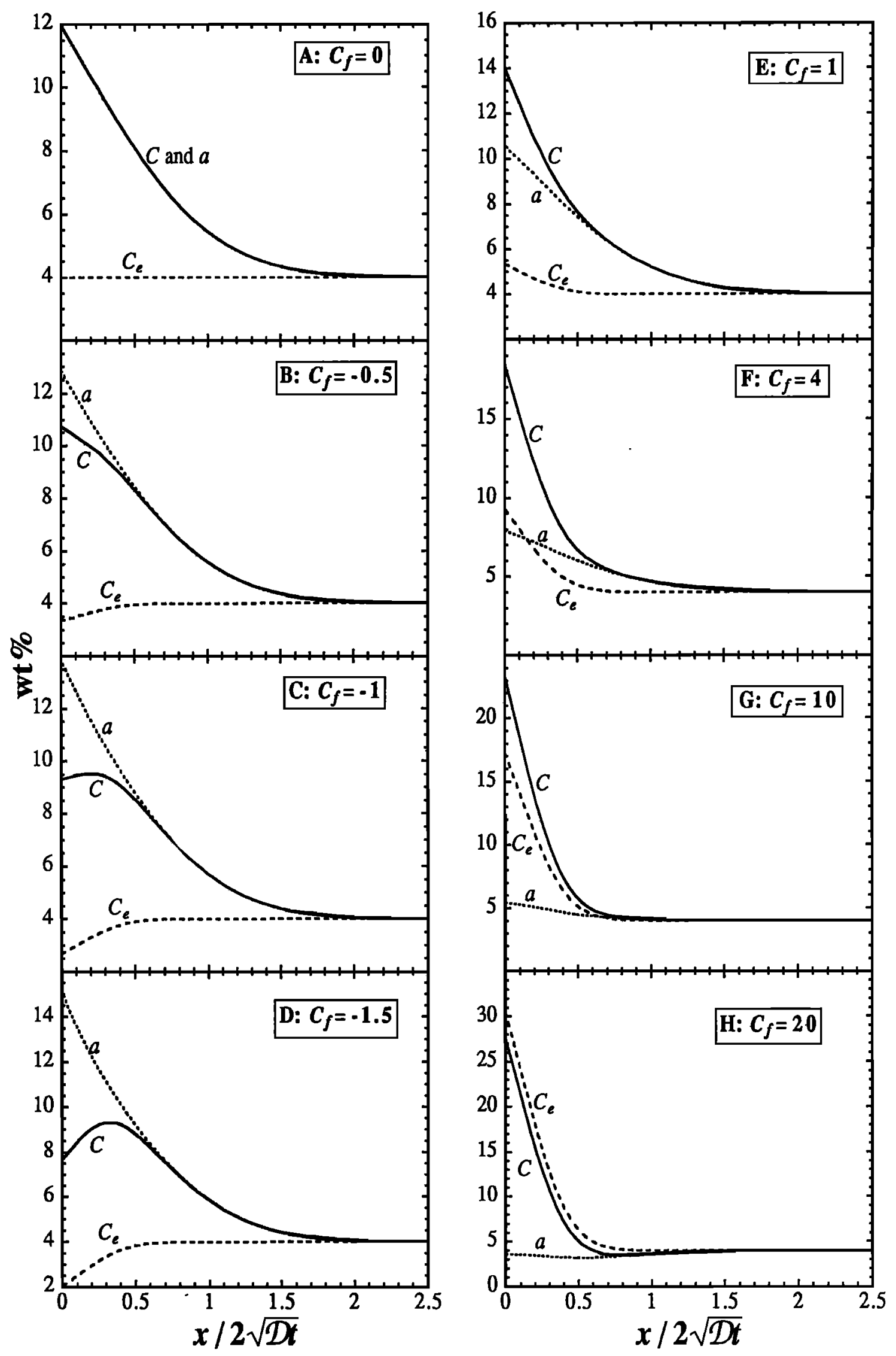

Fig. 6. Calculated $C$ (solid curves), $a$ (dotted curves) and $C_{e}$ (dashed curves) profiles for crystal dissolution for $C_{\infty}=4 \%$ and $C_{s}=50 \%$ with $\mathcal{D} / D_{b}=8$ and $A=0.3 \mu \mathrm{m} \mathrm{s}^{-1 / 2}$. At $x=\infty, a$ is defined to equal $C$. 
These simulation examples illustrate the richness of the solution to the modified effective binary diffusion equation.

\section{Chemical Diffusion During Diffusive Crystal Growth}

The model presented above can be applied to simulate diffusion profiles during crystal growth. If the relevant data are known, other applications are, in principle, straightforward.

Consider $\mathrm{CaO}$ diffusion during olivine growth from an oversaturated silicate liquid. The growth rate is assumed to be diffusion controlled, and hence inversely proportional to $t^{1 / 2}$. The diffusion can be described by (14) to (18) with negative $A$ to account for the opposite direction of boundary motion. The $\mathrm{SiO}_{2}$ and/or $\mathrm{Al}_{2} \mathrm{O}_{3}$ concentrations in the interface melt depend on and can be estimated from the growth rate using $C_{0}=\left(b C_{s}+C_{\infty}\right) /(1+$ b) where $b=\sqrt{\pi} \xi_{\mathrm{e}} \xi^{2} \operatorname{erfc}(-\xi)$, where $\xi=A / \sqrt{D_{b}}$ [Zhang et al., 1989]. The parameter $C_{f}$ depends on and can be estimated from the difference in $\mathrm{Si}+\mathrm{Al}$ between interface and initial melt (see later discussions and Figure 10). Therefore, given $D_{b}, \mathcal{D}$ and $A$, the $\mathrm{CaO}$ concentration profile can be calculated assuming that the $\mathrm{CaO}$ concentration in olivine is zero. Simulation results show that there is always uphill diffusion of $\mathrm{CaO}$, but for $|A| / \sqrt{D_{b}} \leq$ 0.01 , the interface melt composition is nearly the same as the initial melt composition, and the compositional variation in melt near the interface cannot be resolved. Only when $|A| / \sqrt{D_{b}}>$ 0.01 , will there be a measurable concentration profile, which will be monotonic for $\mathrm{SiO}_{2}, \mathrm{MgO}$, etc., but will show uphill diffusion for $\mathrm{CaO}$.

Crystal growth in melts usually occurs while the system is cooling and hence the growth rate is affected by cooling rate and may be constant rather than inversely proportional to $t^{1 / 2}$. This problem is more complicated because the concentration profile evolves with time in a complicated way instead of propagating according to the square root of time. The interface melt composition and hence $C_{f}$ also change with time. Simulation results using this model with appropriate boundary conditions and constant $\mathcal{D}$ (assuming temperature variation is small) show that uphill diffusion always occurs for $\mathrm{CaO}$ during olivine growth, as in the case of diffusion-controlled growth rate at constant temperature. However, whether or not this uphill diffusion profile is resolvable depends on the crystal growth rate $V$, the time $t$ since initial growth, and the diffusivities. Roughly, when $V^{2} t / D_{b} \leq 10^{-4}$, uphill diffusion is not measurable. Cashman [1991] concluded that crystal growth rates are about $10^{-10}$ to $10^{-11}$ $\mathrm{cm} / \mathrm{s}$ in most silicate magmas and therefore concentration profiles near those crystals would be almost constant. Taking $D_{b}=10^{-7}$ to $10^{-9} \mathrm{~cm}^{2} / \mathrm{s}$, a diffusion profile should be measurable when $t$ is greater than 4 months to 3170 years., i.e., when the size of the crystal is greater than $10 \mu \mathrm{m}$ to $1 \mathrm{~cm}$. Convection in the magma may wipe out the diffusion profile. Diffusion profiles (including uphill diffusion profiles) can be resolved only in some rare cases in which crystal dissolution or growth rates are unusually high ( $\geq$ $10^{-8} \mathrm{~cm} / \mathrm{s}$ ), such as during xenolith digestion [e.g., Brearley and Scarfe, 1986].

\section{MODELING DIFFUSION DURING DIFFUSIVE CRYSTAL DisSOlUTION}

The model developed above is now applied to fit diffusion profiles during diffusive crystal dissolution for which extensive data are available [Zhang et al., 1989]. For simplicity, $p, D_{b}, \mathcal{D}$, and $C_{f}$ are assumed to be constant. Of the parameters involved in (14) to (18), $C_{s}$ and $C_{\infty}$ can be directly measured with very small uncertainties, and $A$ can be obtained from the crystal dissolution distance [Zhang et al., 1989] with a small uncertainty. The term $D_{b}$ is taken as the diffusivity of $\mathrm{SiO}_{2}+\mathrm{Al}_{2} \mathrm{O}_{3}$ for alkalis and divalent cation oxides because they show uphill diffusion in either $\mathrm{SiO}_{2}$ and $\mathrm{Al}_{2} \mathrm{O}_{3}$ gradients (olivine dissolution) or an $\mathrm{Al}_{2} \mathrm{O}_{3}$ gradient alone (diopside dissolution); while $\mathrm{D}_{b}$ for $\mathrm{TiO}_{2}, \mathrm{Ce}$, and $\mathrm{V}$ is taken to be the diffusivity of $\mathrm{SiO}_{2}$ because $\mathrm{TiO}_{2}$ uphill diffusion is absent when a $\mathrm{SiO}_{2}$ gradient is absent during diopside dissolution [Zhang et al., 1989] and Ce and V are thought to behave more like $\mathrm{TiO}_{2}$ than the alkalis and divalent cations (also better fits for $\mathrm{Ce}$ and $\mathrm{V}$ are obtained this way). Hence the only free parameters in (14) to (18) are $C_{f}$ and $\mathcal{D}$ (instead of $D$ alone for fitting using a concentration-based effective binary diffusion model). For nonmonotonic profiles, both $\mathcal{D}$ and $C_{f}$ are obtained from the fits. The $\mathrm{CaO}$ and $\mathrm{FeO}$ profiles [Zhang et al., 1989] show less scatter and are considered in great detail here. For monotonic profiles, only the $\mathrm{MgO}$ profile during olivine dissolution is considered to illustrate how one can fit $\mathcal{D}$ by fixing $C_{f}$ using two-liquid partition data. Weight percent concentrations are used since the actual diffusing species are not known. Cation mole percent can be used with similar results; concentrations in terms of mass/volume are also used in the fitting but the results are not significantly better (see discussions following equation (9) for the unit of $C$ ). Note that the two parameters $\left(C_{f}\right.$ and $\left.\mathcal{D}\right)$ have to fit many features in such a profile, including the general shape, the length of the profile, the position and extent of extrema, interface concentration, etc. Least squares fits to experimental data were obtained by minimizing

$$
\chi^{2}\left(\mathcal{D}, C_{f}\right)=\sum_{k}\left[\left(C_{k}-\hat{C}_{k}\right) / \sigma_{k}\right]^{2},
$$

where $C_{k}$ is the measured concentration at point $k, \hat{C}_{k}$ is the numerically calculated concentration based on (14) to (18), $\sigma_{k}$ is the analytical error in the measured concentration, and $\chi^{2}$ is the chi-square, a function of $\mathcal{D}$ and $C_{f}$.

Some fits to experimental concentration profiles are shown in Figure 7. Since $\rho, D_{b}$, and $\mathcal{D}$ are assumed to be constant for these fits, it is expected that in general, the best fits will be obtained if compositional variations across the profile are relatively small. Figures $7 \mathrm{a}$ and $7 \mathrm{~b}$ show fits to $\mathrm{CaO}$ and $\mathrm{FeO}$ profiles during $\mathrm{San}$ Carlos olivine ( Fogo $)$ dissolution into andesitic melts $(56.7 \%$ $\mathrm{SiO}_{2}$ ) at $1185^{\circ} \mathrm{C}$ and $0.5 \mathrm{GPa}$ (run 229). At this low temperature, the initial melt is close to saturation; hence the composition of the interface melt (which is similar to the melt at saturation) is relatively close to that of the initial melt; and compositional variations across the profile are relatively small. The fits are excellent with no systematic misfits. As the experimental temperature increases, the initial melt is farther away from olivine saturation; hence the interface melt composition is more different from that of the initial andesitic melt and compositional variations across the profile are greater. Therefore the quality of the fits is expected to deteriorate. Figures $7 \mathrm{c}$ and $7 \mathrm{~d}$ show fits to profiles at $1250^{\circ} \mathrm{C}$ (run 219) and the fits are very good with only small misfits at the minima. Figures $7 e$ and $7 f$ show fits to profiles at $1285^{\circ} \mathrm{C}$ (run 212), and the fits are good with slightly greater misfits at the minima. Fits to profiles during olivine dissolution into andesitic melt at $1375^{\circ} \mathrm{C}$ and $1.5 \mathrm{GPa}$ (run 236) and $1400^{\circ} \mathrm{C}$ and $0.55 \mathrm{GPa}$ (run 239) show significant and systematic misfits (Table 2 and Figures $7 \mathrm{~g}$ and $7 \mathrm{~h}$ ). The deterioration of the fits with increasing compositional variation across the profile implies that a higher-order model is required to describe the diffusion profile if the compositional variation is large. Among other things, poor fits may reflect compositional dependence of $\rho, D_{b}$, and $\mathcal{D}$, poor approximation of $C_{e}$ by (18), and/or cross terms in (3). Fits using an exponential expression (18a), instead of the linear expression (18), to relate $C_{e}$ and $\mathrm{SiO}_{2}+\mathrm{Al}_{2} \mathrm{O}_{3}$ concentration are not significantly different from those shown in Fig. 7; they are often slightly worse and only 


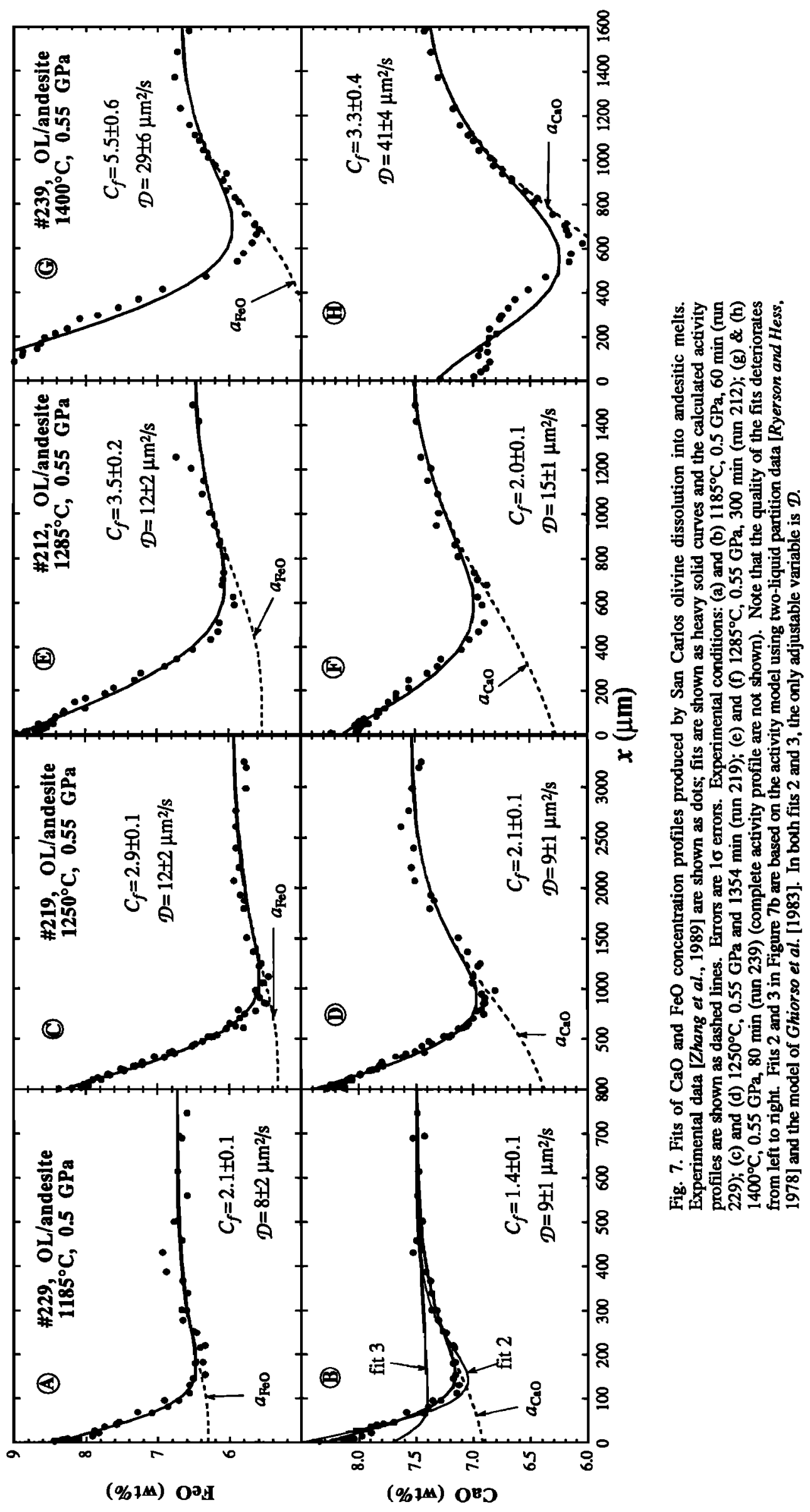


TABLE2. Effective Binary and Intrinsic Effective Binary Diffusivities in Andesitic Melt During Diffusive Crystal Dissolution

\begin{tabular}{|c|c|c|c|c|c|c|c|c|c|c|c|c|c|c|c|c|c|c|}
\hline Run & $\begin{array}{l}T . \\
\cdot \mathrm{C}\end{array}$ & $\begin{array}{c}P, \\
\text { GPa }\end{array}$ & $\begin{array}{c}t, \\
\min \end{array}$ & $\begin{array}{c}A, \\
\mu \mathrm{m} / \sqrt{s}\end{array}$ & $\begin{array}{c}D_{b_{1}} \\
\mu \mathrm{m}^{2} / \mathrm{s} \\
\end{array}$ & $\begin{array}{r}D_{\mathrm{PoO}}, \\
\mathrm{\mu m}^{2} / \mathrm{s} \\
\end{array}$ & $\begin{array}{l}C_{f}^{\mathrm{P} D O} \\
\text { wt } \%\end{array}$ & $\begin{array}{c}S_{\text {FoO, }} \\
\text { wt \% }\end{array}$ & $\begin{array}{l}D_{\mathrm{MgO}} \\
\mu \mathrm{m}^{2} / \mathrm{s}\end{array}$ & $\begin{array}{l}\mathcal{D}_{\mathrm{MgO}}, \\
\mu_{\mathrm{m}}^{2} / \mathrm{s}\end{array}$ & $\begin{array}{c}C_{f}^{\mathrm{M}_{\mathrm{s}} \mathrm{O}}, \\
\mathrm{wt} \%_{0}\end{array}$ & $\begin{array}{l}D_{\mathrm{CaO}} \\
\mu^{2} / \mathrm{s}\end{array}$ & $\begin{array}{l}\text { Deco, } \\
\mu^{2}{ }^{2 / s} \\
\end{array}$ & $\begin{array}{l}C_{f}^{\mathrm{CaO}} . \\
\mathrm{wt} \%\end{array}$ & $\begin{array}{c}\mathrm{SD}_{\mathrm{C} \boldsymbol{O}} \\
\mathrm{wt} \%\end{array}$ & $\begin{array}{r}\mathcal{D}_{\mathrm{K}_{2} \mathrm{O}} \\
{\mu \mathrm{m}^{2} / \mathrm{s}}\end{array}$ & $\begin{array}{l}C_{f}^{\mathrm{K}_{2} \mathrm{O}}, \\
\text { wt \% }\end{array}$ & $\begin{array}{c}S D_{\mathrm{K} 20} \\
\text { wt } \%\end{array}$ \\
\hline \multicolumn{19}{|c|}{ San Carlos Olivine } \\
\hline 229 & 1185 & 0.50 & 60 & 0.063 & 0.7 & $7.8 \pm 2.1$ & 2.1 & 0.11 & 1.9 & $(4.6 \pm 0.4)$ & $(1.0)$ & - & $8.9 \pm 1.1$ & 1.4 & 0.05 & $50 \pm 20$ & -0.53 & 0.022 \\
\hline 219 & 1250 & 0.55 & 1354 & 0.14 & 1.3 & $12.4 \pm 1.6$ & 2.9 & 0.09 & 3.0 & $(6.4 \pm 0.3)$ & (1.4) & 一 & $8.8 \pm 0.6$ & 2.1 & 0.07 & - & - & - \\
\hline $212^{*}$ & 1285 & 0.55 & 300 & 0.23 & 2.9 & $11.8 \pm 2.3$ & 3.5 & 0.15 & 5.2 & $(11.4 \pm 0.9)$ & (1.5) & - & $15.3 \pm 1.3$ & 2.0 & 0.07 & $23 \pm 4$ & -0.76 & 0.036 \\
\hline 222 & 1302 & 0.55 & 139 & 0.32 & 3.5 & $19 \pm 5$ & 3.8 & 0.15 & 6.5 & (14.2 \pm 1.2$)$ & $(1.7)$ & & $25 \pm 3$ & 2 & 0.10 & $61 \pm 18$ & -0.75 & 0.04 \\
\hline 236 & 1375 & 1.5 & 30 & 0.89 & 14.5 & $38 \pm 6$ & 4.0 & 0.15 & 24 & $(44 \pm 3)$ & (1.8) & & $43 \pm 4$ & 2.4 & 0.09 & $163 \pm 38$ & -0.79 & 0.05 \\
\hline 239 & 1400 & 0.55 & 80 & 1.05 & 10.5 & $29 \pm 6$ & 5.5 & 0.23 & 19 & $(39+3)$ & (2.5) & & $41 \pm 4$ & 3.3 & 0.12 & $200 \pm 64$ & -1.01 & 0.08 \\
\hline \multicolumn{19}{|c|}{ Pure Synthetic Forsterite } \\
\hline 227 & 1300 & 0.55 & 150 & 0.25 & 3.0 & $13.3 \pm 1.2$ & 4.0 & 0.09 & 6.5 & $(15.4 \pm 1.5)$ & $(1.7)$ & - & $25 \pm 3$ & 1.9 & 0.08 & $69 \pm 21$ & -0.74 & 0.036 \\
\hline 228 & 1300 & 0.50 & 40 & 03 & 5.0 & $14.8 \pm 1.5$ & 4.5 & 0.14 & 9.1 & $(19.7 \pm 0.9)$ & $(2.0)$ & & $20.2 \pm 1.3$ & 2.6 & 0.08 & - & 一 & 一 \\
\hline \multicolumn{19}{|c|}{ Diopside } \\
\hline 246 & 1285 & 0.55 & 70 & 0.68 & 3.4 & $11.9 \pm 1.6$ & 5.2 & 0.16 & 4.4 & - & 一 & 9.9 & 一 & 一 & 一 & 一 & 一 & 一 \\
\hline 223 & 1305 & 1.05 & 70 & 0.37 & 3.0 & $16.0 \pm 1.0$ & 2.8 & 0.06 & 4.3 & & 一 & 8.6 & - & - & - & - & - & - \\
\hline \multicolumn{19}{|c|}{ Quor } \\
\hline 234 & 1300 & 0.50 & 60 & 0.11 & 0.28 & - & - & - & 0.20 & $\rightarrow$ & - & 0.21 & - & - & - & $42 \pm 23$ & 0.75 & 0.052 \\
\hline
\end{tabular}

$D$ (effective binary diffusivity) values are from Zhang et al. [1989]. $\mathcal{D}$ (intrinsic effective binary diffusivity) values are obtained by fitting uphill diffusion profiles. When $C_{f}$ is fixed in the fitting, $D$ and the fixed $C_{f}$ are given in parentheses. SD is standard deviation in concentration (wt $\varphi_{0}$ ) in fitting the concentration profile equal to $\bar{\sigma} \sqrt{\chi^{2} /(n-m)}$, where $n$ is the number of data points, $m(=2)$ is the number of parameters in fitting, and $\bar{\sigma}$ is average error of analysis. Errors are given at $1 \sigma$ level. They are nominal fitting errors and do not include errors associated with $A, D_{b}$. etc.

- $D_{\mathrm{N}_{2} \mathrm{O}}=14 \pm 3 ; D_{\mathrm{ce}}=4.3 \pm 1.1 ; D_{\mathrm{TiO}_{2}}=1.4 \pm 0.5 ; D_{\mathrm{v}}=0.9 \pm 0.2 \mu \mathrm{m}^{2} / \mathrm{s}$.

occasionally slightly better. Introducing a compositional dependence in $D_{b}$ and $\mathcal{D}$ generates better fits, but this would require introducing more parameters in least squares fittings. Compositional dependence of density $\rho$ is difficult to treat quantitatively. Such considerations are beyond the scope of this paper. Only diffusion profiles at or below $1300^{\circ} \mathrm{C}$ which are well fit using constant $D_{b}$ and $\mathcal{D}$ are modeled using this treatment.

Figure 8 shows fits to $\mathrm{TiO}_{2}, \mathrm{MgO}, \mathrm{Na}_{2} \mathrm{O}, \mathrm{K}_{2} \mathrm{O}, \mathrm{Ce}$, and $\mathrm{V}$ concentration profiles produced by $\mathrm{San}$ Carlos olivine dissolution into andesitic melt (run 212; Figures 7e and 7f show fits to $\mathrm{FeO}$ and $\mathrm{CaO}$ for this experiment). All the fits are good, although $\mathcal{D}$ and $C_{f}$ values are not well constrained for the alkalis as discussed earlier in the simulation section. The intrinsic diffusivities seem to decrease with increasing valence though the trend is not perfect, with $\mathcal{D}_{\mathrm{V}}\left(\mathrm{V}^{3+}\right)$ and $\mathcal{D}_{\mathrm{TiO}_{2}}\left(\mathrm{Ti}^{4+}\right)$ being the smallest and smaller than $D_{b}$. One might intuitively think that the position of the extreme is proportional to $\mathcal{D}^{1 / 2}$. However, this is not the case. The position of the minimum depends not only on $\mathcal{D}$ but also on $C_{f}$, as demonstrated by Figures 7 and 8 . Hence $\mathcal{D}_{\mathrm{V}}<\mathcal{D}_{\mathrm{TiO}_{2}}$ even though the minimum on the $\mathrm{TiO}_{2}$ profile is at a smaller distance away from the olivine-melt interface. Figure 8 also includes a fit to the monotonic $\mathrm{MgO}$ concentration profile in which $C_{f}$ is estimated from two-liquid partition coefficient [Ryerson and Hess, 1978] and fixed. Only $\mathcal{D}_{\mathrm{M}_{\mathrm{B}} \mathrm{O}}$ is adjustable in fitting this and other $\mathrm{MgO}$ profiles. The fit is quite good, and the resultant $\mathcal{D}_{\mathrm{MgO}}$ is similar to $\mathcal{D}_{\mathrm{FeO}}$ and $\mathcal{D}_{\mathrm{CaO}}$.

Figures $9 \mathrm{a}$ and $9 \mathrm{~b}$ show fits to $\mathrm{FeO}$ and $\mathrm{CaO}$ profiles produced by pure synthetic forsterite dissolution (run 228). FeO uphill diffusion is better illustrated in this case than in the case of San Carlos olivine dissolution because the synthetic forsterite contains a negligible amount of $\mathrm{FeO}$ so the enrichment of $\mathrm{FeO}$ toward the interface clearly shows $\mathrm{FeO}$ diffuses uphill toward the interface melt. Figure $9 \mathrm{c}$ shows fit to $\mathrm{K}_{2} \mathrm{O}$ profile produced during quartz dissolution into andesitic melt (run 234). The $\mathcal{D}_{\mathrm{K}_{2} \mathrm{O}}$ value based on fitting concentration profile during quartz dissolution agrees with that during olivine dissolution. Other concentration profiles produced by quartz dissolution into the andesitic melt appear to be monotonic but anomalously short with $D$ values close to $D_{b}$. Simulation results of $\mathrm{MgO}, \mathrm{CaO}$, and $\mathrm{FeO}$ profiles produced by quartz dissolution using $C_{f}$ based on twoliquid partition data and $\mathcal{D}$ for profiles produced by olivine dissolution show that these profiles can be matched very well. Figure 9d compares the calculated $\mathrm{CaO}$ profile and the experimental data. The good agreement shows that $\mathcal{D}_{\text {cao }}$ during quartz dissolution into andesitic melt (in which the interface composition is rhyolitic and $D_{\mathrm{CaO}}=0.22 \mu \mathrm{m}^{2} / \mathrm{s} \approx D_{b}=0.28$ $\mu \mathrm{m}^{2} / \mathrm{s}$ ) is similar to that during olivine dissolution (in which the interface composition is basaltic and $D_{b}=3.0 \mu \mathrm{m}^{2} / \mathrm{s}<D_{\mathrm{CaO}}=20$ $\left.\mu \mathrm{m}^{2} / \mathrm{s}\right)$. Therefore it appears that, unlike $D$ values, the intrinsic effective binary diffusivities are not strongly dependent on melt composition. Figures $9 \mathrm{e}$ and $9 \mathrm{f}$ show fits to uphill $\mathrm{FeO}$ diffusion profiles during diopside dissolution (runs 223 and 246). All of these fits are very good.

Table 2 reports results for more fits. The $\mathcal{D}$ values obtained for different experiments (dissolution of different minerals) at similar temperature and pressure are similar. In general, $\mathcal{D}$ values from fits to alkali profiles have large errors because (1) the quality of data is lower (most $\mathrm{Na}_{2} \mathrm{O}$ and a few $\mathrm{K}_{2} \mathrm{O}$ profiles are not good and are not used to fit); (2) $\mathcal{D} \gg D_{b}$ (in the extreme case when $\mathcal{D}$ $\gg D_{b}$, quasi-equilibrium is reached and it is not possible to obtain $\mathcal{D}$ from concentration profiles); and (3) $\mathcal{D}$ values are inherently not well constrained by these profiles as shown in the simulations in a previous section.

Given the two major approximations embodied in (14) and (18) and the assumption of constant $p, D_{b}$, and $\mathcal{D}$, it is remarkable that such a simple model fits the experimental diffusion profiles so well. There are many factors that could lead to poor fits, such as significant cross terms, discrepancies in the $C_{e}$ expression, and variable $D_{b}$ and/or $\mathcal{D}$. The good fits obtained for many examples suggest that this model still captures the essence of the problem, although limitations of the model are evidenced by the systematic misfits when the compositional variation across the profile is too large. Variable $D_{b}$ and/or $\mathcal{D}$ can be incorporated into the model in the future if the compositional dependence can be specified.

\section{Discussions AND APPLICATIONS}

\section{Comparison With Two-Liquid Partition Coefficients and Results From Solution Models}

To examine whether the activity model employed to fit experimental profiles (i.e., equation (18)) is meaningful, the model results are compared with previous experimental results on two-liquid partitioning [Ryerson and Hess, 1978] and a thermodynamic mixing model for silicate melts [Ghiorso et al., 1983]. A hypothetical two-liquid partition coefficient $(K)$ between the interface melt and the melt at $x=\infty$ during crystal dissolution is calculated from $C_{f}$ values obtained in this study by 

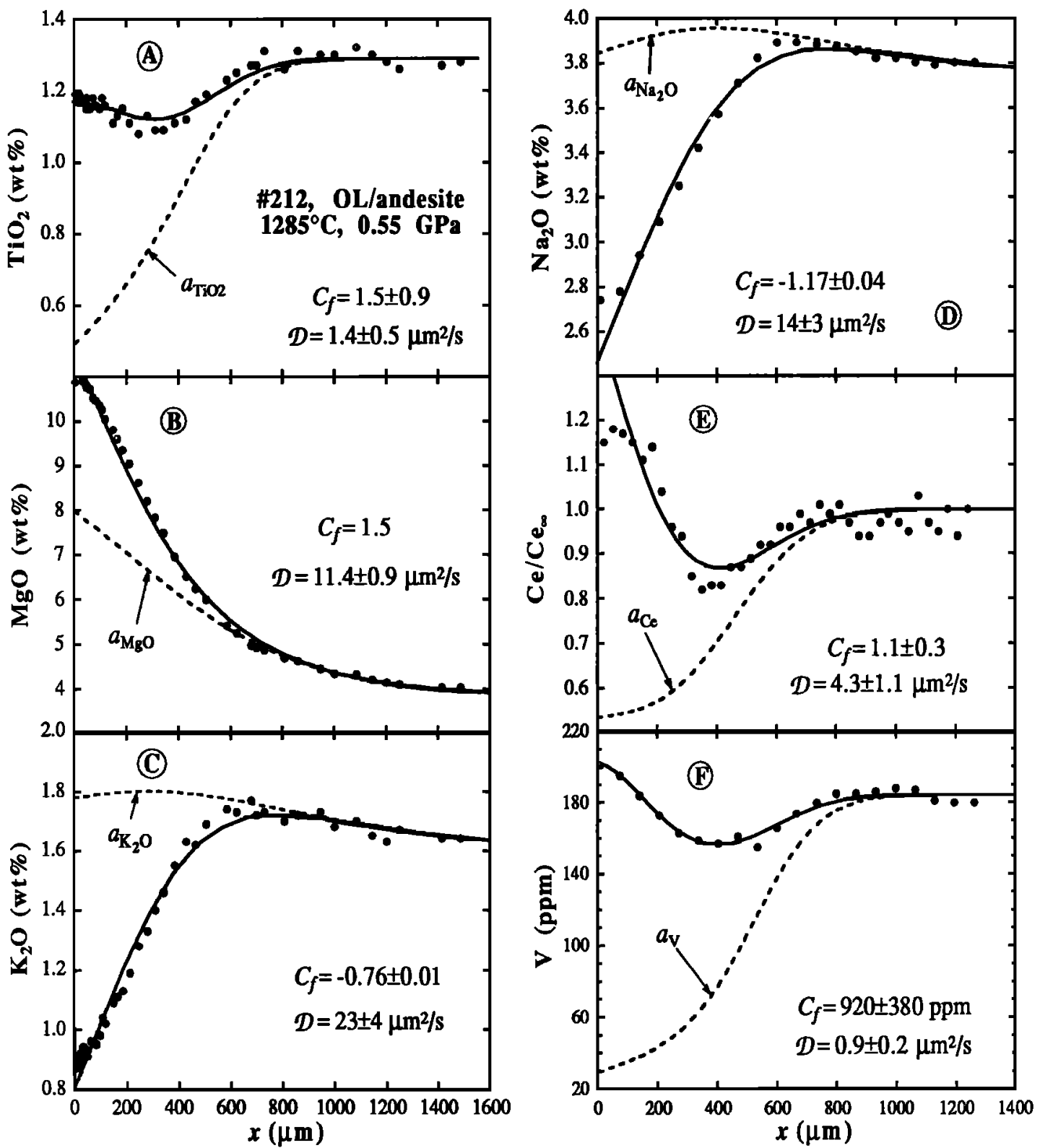

Fig. 8. Fits of $\mathrm{TiO}_{2}, \mathrm{MgO}, \mathrm{K}_{2} \mathrm{O}, \mathrm{Na}_{2} \mathrm{O}, \mathrm{Ce}$, and $\mathrm{V}$ concentration profiles produced by $\mathrm{San}$ Carlos olivine dissolution into andesitic melts at $1285^{\circ} \mathrm{C}$ and $0.55 \mathrm{GPa}$ (nin 212). $\mathrm{Na}_{2} \mathrm{O}, \mathrm{Ce}$, and $\mathrm{V}$ are measured by ion microprobe. Absolute concentration of $\mathrm{Ce}$ is not known. Experimental data are shown as dots, fits are shown as heavy solid curves, and the calculated activity profiles are shown as dashed lines.

$K=C_{e} d_{x=0} / C_{e} d_{x=\infty}=1+\left(C_{f} / C_{\infty}\right) \operatorname{erfc}\left(-A / \sqrt{D_{b}}\right)$, which can then be compared to true two-liquid partition coefficients $K$ from previous experimental studies (assuming that they mean the same thing). Unfortunately, a direct comparison is not possible because the diffusion and partitioning studies were performed at different temperatures and pressures and on different bulk melt compositions. It is therefore necessary to specify how two-liquid partition coefficients vary with temperature, pressure, and bulk composition. This is a complicated issue, but a simple approximation is to ignore the temperature and pressure dependence and to relate two-liquid partition coefficient $K$ to the difference in $\mathrm{Si}+\mathrm{Al}$ cation mole percent (which characterizes the degree of polymerization of the melt) between the two liquids (i.e., ignoring the dependence of $K$ on concentrations of other components) [Lesher, 1986; Jones and Mavin, 1990]. In Figure 10 , values of $\ln K$ calculated from fits of diffusion profiles are compared to the experimental two-liquid partition data for different end-member compositions [Ryerson and Hess, 1978]. Considering the rough parameterization which makes this comparison possible, the agreement between $K$ values derived from this model and $K$ values determined experimentally is very good. The overall agreement suggests that the $C_{f}$ parameter obtained by fitting the concentration profiles in this study is physically meaningful and that the concept of two-liquid partitioning [Watson, 1982], which is pivotal to this model, is useful. Note that $\ln K_{\mathrm{FeO}}$ is slightly but systematically greater than $\ln K_{\mathrm{CaO}}$ based on this study, but experimentally determined $K_{\mathrm{FeO}}$ and $K_{\mathrm{CaO}}$ are similar. The $K$ values for $\mathrm{K}_{2} \mathrm{O}$ derived from this model are somewhat greater than experimentally determined $K$ values, likely due to large errors in the $K$ values for $\mathrm{K}_{2} \mathrm{O}$, as discussed earlier.

Another way to examine whether the activity model employed in this paper is meaningful is to compare directly the activity coefficients based on model fitting results to those based on two- 

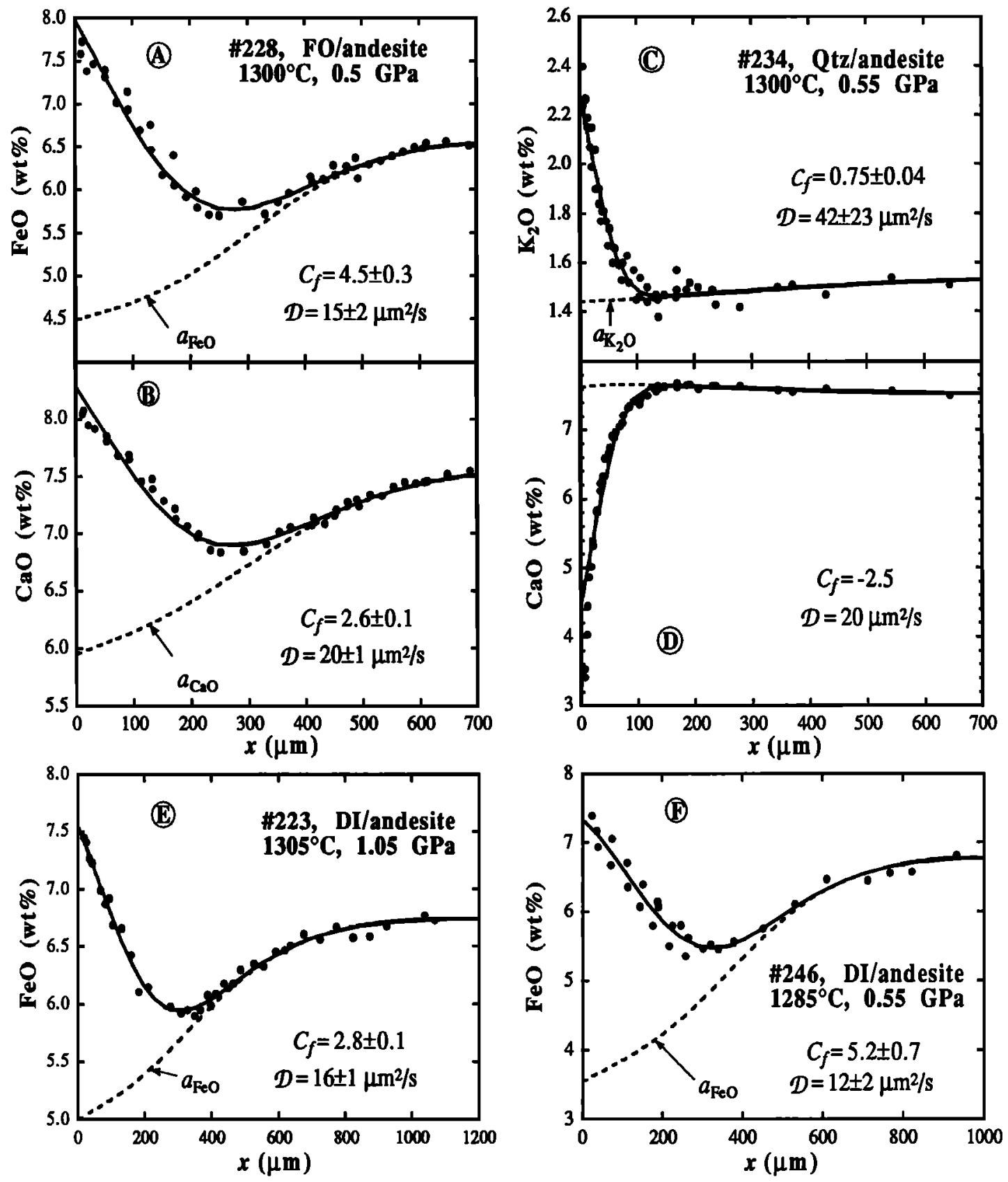

Fig. 9. Fits of concentration profiles during dissolution of pure forsterite, quartz, and diopside into andesitic melts. Experimental data are shown as dots, fits are shown as heavy solid curves, and the calculated activity profiles are shown as dashed lines. (a) and (b) $\mathrm{CaO}$ and $\mathrm{FeO}$ concentration profiles produced by synthetic pure forsterite dissolution (run 228). (c) and (d) $\mathrm{K}_{2} \mathrm{O}$ and $\mathrm{CaO}$ profiles produced by quartz dissolution (nun 234). Note that the activity profiles for $\mathrm{K}_{2} \mathrm{O}$ and $\mathrm{CaO}$ are almost constant, suggesting quasi-equilibrium distribution is almost reached because $\mathcal{D}_{\mathrm{K}_{2} \mathrm{O}}$ and $\mathcal{D}_{\mathrm{co}} \gg D_{b}$. (e) and (f) $\mathrm{FeO}$ concentration profile produced by diopside dissolution into andesitic melt (runs 223 and 246).

liquid partition experiments or those based on thermodynamic mixing models. The three methods of calculating activity coefficients at any point along a diffusion profile (Figure 11) are as follows:

1. Based on this model, the activity coefficient of a component is inversely proportional to $C_{e}$. Defining $\gamma=1$ for the initial melt, then $\gamma=C_{\infty} / C_{e}$

2. Since the two-liquid partition coefficients $K=C_{\operatorname{liq} 2} / C_{\text {liq } 1 \text {, }}$ and since at equilibrium $a_{\text {liq } 2}=a_{\text {liq } 1}$ in the two immiscible liquids, $\gamma_{\text {liq } 2} / \gamma_{\text {liq } 1}=(a / C)_{\text {liq } 2} /(a / C)_{\text {liq } 1}=1 / K$. Since experimental two-liquid partition data show that $\ln K$ is roughly a linear function of $\Delta(\mathrm{Si}+\mathrm{Al}$ ) (Figure 10), the $K$ value (hence $\gamma$ ratio) between any two liquids can be calculated given difference in $\mathrm{Si}+\mathrm{Al}$ between the two liquids. Defining $\gamma=1$ for the initial melt, $\gamma$ at any point along a diffusion profile can be calculated from two-liquid partition data parameterized in Figure 10.

3. Activity coefficient for a given component at any point along a diffusion profile can be directly calculated from the mixing model of Ghiorso et al. [1983]. Dividing the activity coefficient at any point by that of the initial melt, the result is the $\gamma$ with respect to the initial melt.

Figure 11 a compares $\gamma_{\mathrm{CaO}}$ values in run 229 calculated from this model, those calculated from two-liquid partition coefficients, and those from the mixing model of Ghiorso et al. 


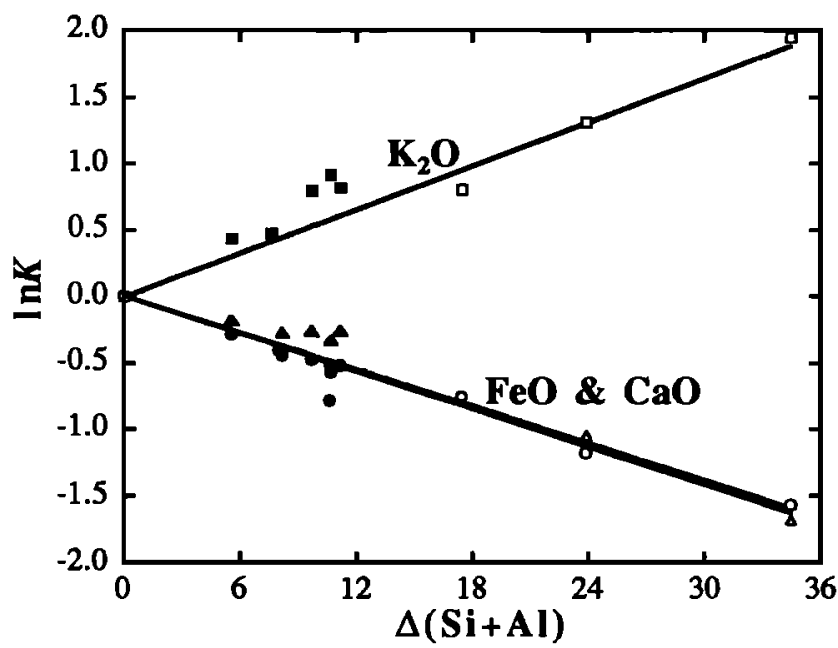

Fig. 10. Comparison of calculated $K$ values from this study (solid symbols) to those measured in two-liquid partition experiments (open symbols; data from Watson [1976] and Ryerson and Hess [1978]) in a InK versus $\Delta(\mathrm{Si}+\mathrm{Al})$ plot. $\Delta(\mathrm{Si}+\mathrm{Al})$ represent the difference in $\mathrm{Si}+\mathrm{Al}$ cation mole percent between the two end-member liquids in crystal dissolution experiments or the two liquids used in the partitioning experiments. Fits to two-liquid partition data (not the $K$ values from this study) are shown as solid lines. Fits are forced to pass through the origin (i.e., $K$ must be unity when the two liquids have identical compositions). Squares, $\mathrm{K}_{2} \mathrm{O}$; circles, $\mathrm{FeO}$; and triangles, $\mathrm{CaO}$.

[1983]. The model results are similar to those based on twoliquid partition experiments but different from those from the mixing model of Ghiorso et al. [1983], which has some problems in reproducing two-liquid partition data for $\mathrm{CaO}$ and a few other components. To check the validity of this comparison, activities calculated from two-liquid partition data and from the solution model of Ghiorso et al. [1983] are also used to fit $\mathrm{CaO}$ concentration profiles for run 229 using the procedure described before with $\mathcal{D}$ being the only adjustable parameter. The fits are shown in Figure $7 \mathrm{~b}$ (fits 2 and 3). The fit using activity from two-liquid partition data (fit 2 ) is acceptable but the one using the solution model of Ghiorso et al. (fit 3 ) is totally off, consistent with comparisons in Figure $11 \mathrm{a}$. Figure $11 \mathrm{~b}$ compares $\gamma_{\mathrm{FeO}}$ values derived from run 212 (olivine dissolution into andesitic melt) with those derived from run 246 (diopside dissolution into andesitic melt), the only two experiments with similar temperature and pressure but different compositional gradients. During diopside dissolution into andesitic melt, the Si content is nearly constant so that variations in $\mathrm{Si}+\mathrm{Al}$ are almost entirely due to variations in $\mathrm{Al}$ content, whereas during olivine dissolution into andesitic melt, both $\mathrm{Si}$ and $\mathrm{Al}$ decrease toward the olivinemelt interface. The $\gamma_{\mathrm{FeO}}$ values as a function of $\mathrm{Si}+\mathrm{Al}$ calculated from this work agree within error for the two experiments (Figure 11b). Therefore the activity coefficient largely depends on $\mathrm{Si}+\mathrm{Al}$ cation mole percent at least for $\mathrm{FeO}$, instead of either $\mathrm{Si}$ or $\mathrm{Al}$. This result shows internal consistency of the assumptions adopted earlier. When compared with $\gamma_{\mathrm{FeO}}$ values from two-liquid partition data and from the model of Ghiorso et al. [1983], they all agree very well (Figure 11b), demonstrating that the activity coefficients obtained with this model are physically meaningful.

\section{Comparisons Between $\mathcal{D}$ values of Different Components and Between $\mathcal{D}$ and $D$}

Figure 12 plots $\mathcal{D}$ values of different components (obtained from this study) and $D_{\mathrm{MgO}}$ and $D_{\mathrm{CaO}}$ values [Zhang et al., 1989] in an Arrhenius diagram. $\mathcal{D}_{\mathrm{MgO}}$ values shown in Figure 12 are obtained using the model fit by fixing $C_{f}$ which is estimated from two-liquid partition coefficients. It is interesting to note that $\mathcal{D}_{\text {CaO }}$ is about $40 \%$ greater than $\mathcal{D}_{\mathrm{MgO}}$, while $\mathcal{D}_{\text {FeO }}$ is between $\mathcal{D}_{\mathrm{MgO}}$ and $\mathcal{D}_{\mathrm{CaO}}$ in this temperature range. $\mathcal{D}$ values of divalent components, $\mathrm{FeO}, \mathrm{MgO}$, and $\mathrm{CaO}$, are within a factor of 2 . On the other hand, intrinsic diffusivities of $\mathrm{K}_{2} \mathrm{O}$ are higher than those of the divalent components, even though the error for $\mathcal{D}_{\mathrm{K}_{2} \mathrm{O}}$ is very large. Values of $\mathcal{D}_{\mathrm{K}_{2} \mathrm{O}}$ derived from quartz dissolution experiment (in which the interface melt is rhyolitic) and olivine dissolution experiment (in which the interface melt is basaltic) are similar. As discussed earlier, $\mathcal{D}$ values of $\mathrm{CaO}, \mathrm{MgO}$ and FeO from olivine dissolution experiments can be used to predict concentration profiles during quartz dissolution. Therefore it appears that intrinsic effective binary diffusivities are less dependent on bulk compositions or directions of diffusive paths than concentration-based effective binary diffusivities. The $\mathcal{D}$ values seem to decrease with increasing valence of the
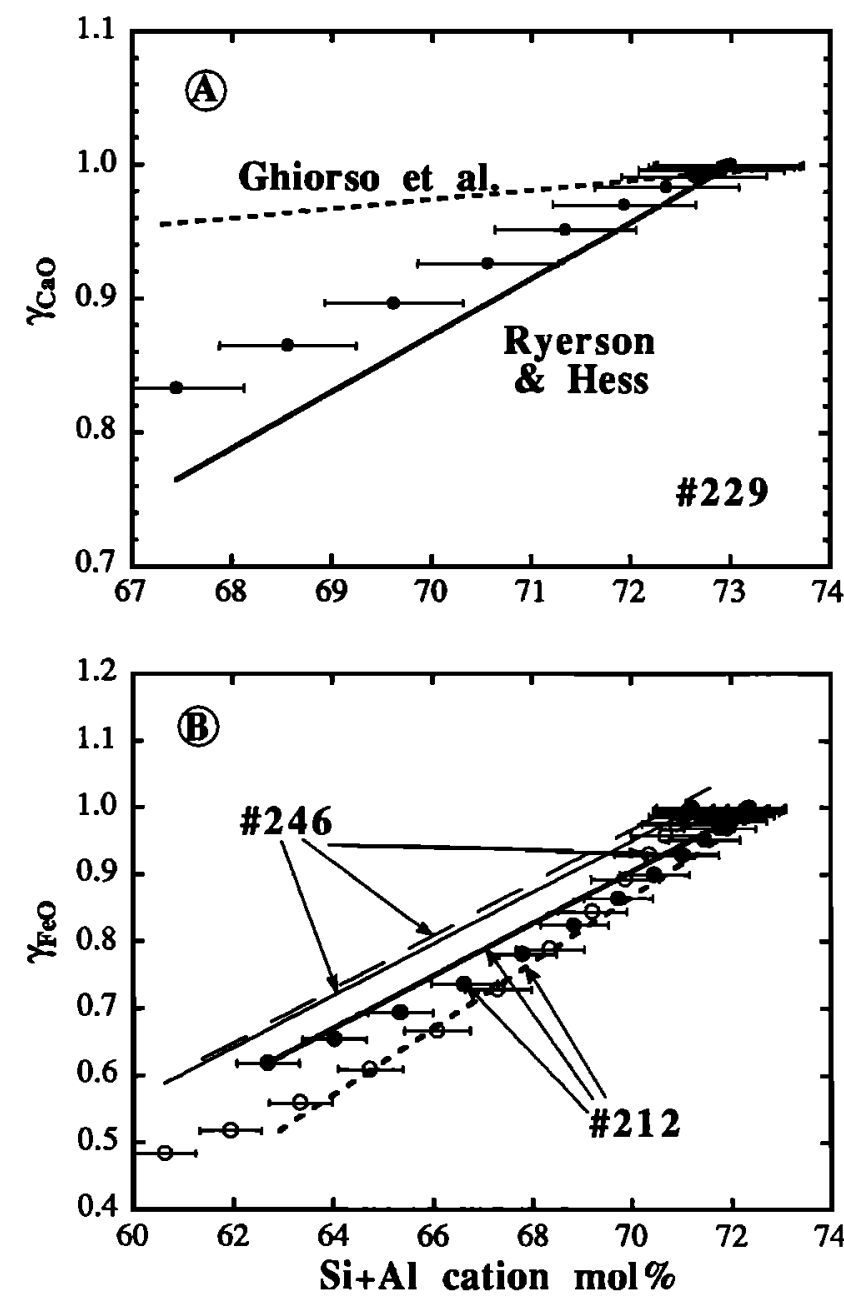

Fig. 11. Calculated activity coefficients vs. Si+Al cation mole percent from fitting results in this study (open and filled dots) compared to those from two-liquid partition coefficients of Ryerson and Hess [1978] as parameterized in Figure 10 and those from the mixing model of Ghiorso et al. [1983]. Uncertainties in $\mathrm{Si}+\mathrm{Al}$ are assumed to be $1 \%$ relative. Uncertainties in $\gamma$ values are difficult to estimate and are hence not shown. $\gamma$ in the initial melt is defined to be 1. (a) $\gamma_{\mathrm{CrO}}$ in nun 229. Solid line is based on work by Ryerson and Hess [1978]; and dashed line is based on work by Ghiorso et al. [1983]. (b) $\gamma_{\mathrm{FeO}}$ in nun 212 and $246\left(1285^{\circ} \mathrm{C}, 0.55 \mathrm{GPa}\right)$. Solid dots for run 212 and open dots for run 246. Calculated $\gamma_{\mathrm{FeO}}$ values based on Ryerson and Hess [1978] are shown as heavy solid line for run 212 and light solid line for run 246. Calculated $\gamma_{\mathrm{FeO}}$ values based on Ghiorso et al. [1983] are shown as short dashed line for run 212 and long dashed line for run 246. 


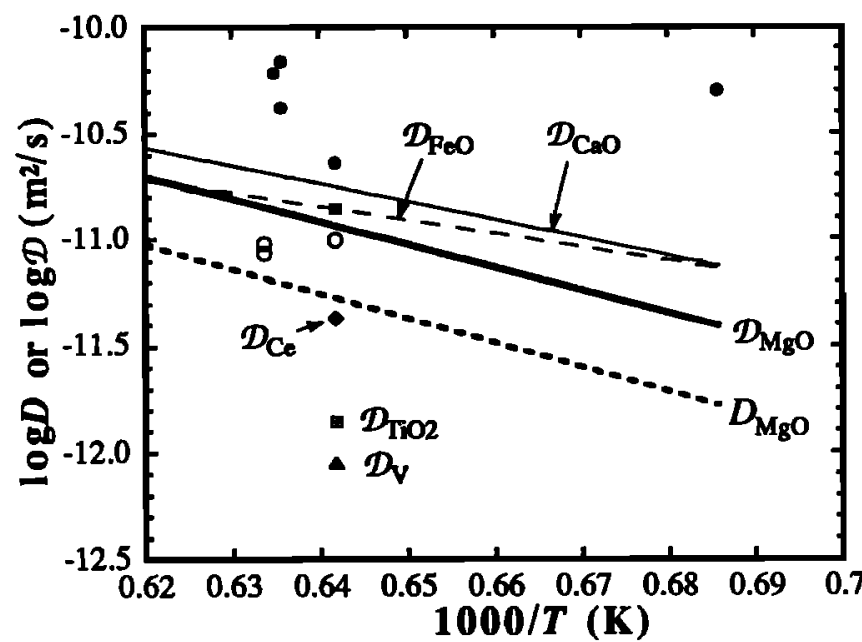

Fig. 12. The $\log D$ and $\log D$ versus $1000 / T$ in an Arrhenius plot. Best fit line for $D_{\mathrm{M}_{0}}$ [Zhang et al., 1989] is shown as a reference. Best fit lines for $\mathcal{D}_{\mathrm{Mg}}$ (obtained by fixing $\left.C_{f}\right), \mathcal{D}_{\mathrm{FeO}}$, and $\mathcal{D}_{\mathrm{CaO}}$ are shown. On average, $\mathcal{D}_{\mathrm{MgO}}=2 D_{\mathrm{MgO}}$. These data points and all errors are not shown so that the figure is readable. Open circles, $D_{\mathrm{coo}}$; solid circles, $\mathcal{D}_{\mathrm{k}, 0}$ (no best fit line is shown due to large errors); solid square, $\mathcal{D}_{\mathrm{Ne}_{2}} \mathrm{O}_{\text {; }}$ solid diamond, $\mathcal{D}_{\text {ce; }}$ solid triangle, $\mathcal{D}_{\mathrm{V}}$ and plus in square, $\mathcal{D}_{\mathrm{TIO}}$.

component. It seems also that $\mathcal{D}$ values of components with the same valence increase slightly with increasing ionic radius (e.g., $\mathcal{D}_{\mathrm{K}_{2} \mathrm{O}}>\mathcal{D}_{\mathrm{Na}_{2} \mathrm{O}} ; \mathcal{D}_{\mathrm{CaO}}>\mathcal{D}_{\mathrm{MgO}_{\mathrm{O}}}$ ), which is counterintuitive. When concentration-based and intrinsic effective binary diffusivities are compared, $\mathcal{D}_{\mathrm{CaO}} \approx 2 D_{\mathrm{CaO}}$ and $\mathcal{D}_{\mathrm{MgO}} \approx 2 D_{\mathrm{MgO}}$ during olivine dissolution and $\mathcal{D}>>D_{b} \approx D$ (for $\mathrm{CaO}, \mathrm{FeO}$, and $\mathrm{MgO}$ ) during quartz dissolution. This is consistent with my earlier discussions that concentration-based effective binary diffusivities are most likely smaller than intrinsic effective binary diffusivities.

\section{Predicting the Direction of Diffusion in Experiments}

Given initial and boundary conditions, concentration profiles can be calculated and the direction of diffusion (occurrence or absence of uphill diffusion) can be predicted based on this model and two-liquid partitioning data. The occurrence of uphill diffusion in the experiments of Zhang et al. [1989] is quantitatively modeled above, and the results support this model. This model also fits well the uphill diffusion profile of $\mathrm{CaO}$ in a diffusion couple of van der Laan et al. (submitted manuscript). Baker [1990] did not observe uphill diffusion for any component during his dacite-rhyolite diffusion couple experiments. This model predicts such absences for all components for the starting composition of Baker [1990] except for $\mathrm{Na}_{2} \mathrm{O}$. $\mathrm{Na}_{2} \mathrm{O}$ is predicted to show a weak uphill diffusion with the difference between maximum and minimum being $0.2 \mathrm{wt} \%$. However, such uphill diffusion may not be resolvable because $0.2 \%$ is less than the size of symbols plotted by the author (Figure 1a of Baker [1990]) and may be within the analytical error of $\mathrm{Na}_{2} \mathrm{O}$. Therefore this model is able to predict the direction of diffusion (the occurrence or absence of uphill diffusion) in controlled experiments.

The model may also be used to predict whether or not a $D$ value should be similar to $D_{b}$ for some published experiments when there is no uphill diffusion (given $D_{b}$ and $\mathcal{D}, D$ can be obtained using a procedure similar to that used in Figure 3 ). The $D$ values for olivine dissolution experiments reported by Zhang $e t$ $a l$. [1989] are predicted to be close to $\mathcal{D}$, rather than to $D_{b}$. This is consistent with the hierarchy observed for these $D$ values $\left(D_{\mathrm{CaO}} \approx 2 D_{\mathrm{MgO}_{8}} \approx 4 D_{\mathrm{Al}_{2} \mathrm{O}_{3}} \approx 6 D_{\mathrm{SiO}_{2}}\right)$. The $D$ values for quartz dissolution experiments reported by Zhang et al. [1989] are predicted to be close to $D_{b}$, rather than to $\mathcal{D}$. This is also consistent with experimental data. For the experiments of Baker [1990], the model predicts that $D$ should be close to $\mathcal{D}$ rather than to $D_{b}$. However, Baker [1990] observed that for most components, $D \approx D_{b}$ (i.e., $D_{\mathrm{CaO}} \approx D_{\mathrm{MgO}_{8}} \approx D_{\mathrm{AbO}_{3}} \approx D_{\mathrm{SiO}_{2}}$ ). The origin of the disagreement is not clear at present but may be due to either one or a combination of the following: (1) a coincidence that in such melts $\mathcal{D}_{\mathrm{C} \mathrm{O}} \approx \mathcal{D}_{\mathrm{MgO}_{\mathrm{O}}} \approx \mathcal{D}_{\mathrm{Al}_{2} \mathrm{O}_{3}} \approx \mathcal{D}_{\mathrm{SiO}_{2}}$, (2) convection in his experimental charge, and (3) large uncertainties in his $D$ values (e.g., a worst case can be found in three duplicate traverses of the same experimental charge $D-34$ which gave three different values of $D_{\mathrm{A}_{2} \mathrm{O}_{3}}$ at $65 \% \mathrm{SiO}_{2}$ to be $0.17,0.022$, and $0.53 \mu \mathrm{m}^{2} / \mathrm{s}$, differing by a factor of 24; see Table 2 of Baker [1990]).

\section{Testing the Validity of the Modified Effective Binary Diffusion Model}

The model developed above can be tested in two ways. The first method involves measuring concentration-based effective binary diffusivities for a component from diffusion couple experiments at a single temperature, pressure, and bulk composition. Let $\mathrm{SiO}_{2}$ and $\mathrm{Al}_{2} \mathrm{O}_{3}$ concentrations of the two halves of the diffusion couple be different and roughly fixed so that $C_{f}$ is nonzero and roughly constant. Let the initial concentration distribution across the couple for the component in consideration (i.e., $\Delta C$ ) vary. Determine the resultant $D$ values. This model predicts that the $D$ values depend on the initial concentration distribution of the component and the dependence is quantitatively predictable. The second method would be to measure both concentration and activity profiles from diffusion experiments, which would allow a direct and rigorous test of this model.

\section{CONCLUSIONS}

An activity-based effective binary diffusion model is presented for diffusion in multicomponent silicate melts. The diffusive flux of a component is assumed to be proportional to the activity gradient of the component (instead of the concentration gradient). The activity coefficient of a component is inversely proportional to its concentration at quasi-equilibrium distribution using the two-liquid partition concept. The quasi-equilibrium distribution is assumed to be a linear or exponential function of $\mathrm{SiO}_{2}$ or $\mathrm{SiO}_{2}+\mathrm{Al}_{2} \mathrm{O}_{3}$ concentration. Assuming a constant concentrationbased effective binary diffusivity for $\mathrm{SiO}_{2}$ or $\mathrm{SiO}_{2}+\mathrm{Al}_{2} \mathrm{O}_{3}$, the quasi-equilibrium concentration is a simple function of distance and time. Assuming a constant intrinsic effective binary diffusivity for the component, the model fits well both the monotonic and uphill diffusion profiles of both major and trace components generated by crystal dissolution experiments when the total compositional variation across the whole profile is small. It appears that, unlike the concentration-based effective binary diffusivities, intrinsic effective binary diffusivities are not very sensitive to the bulk composition or the direction of diffusion. Activity coefficients extracted from the fits agree with those based on two-liquid partition experiments and, in some cases, agree with those based on a regular solution mixing model. The model can also (1) predict the unusually short concentration profiles generated during quartz dissolution into an andesitic melt, (2) predict the direction of diffusion, i.e., the occurrence or absence of uphill diffusion in experiments, and (3) simulate development of concentration and isotopic ratio profiles during diffusion. The success of this simple model suggests that it still captures the essence of the problem. However, I emphasize that the model is not perfect and should be applied only when a more precise approach (such as approaches using diffusivity matrix or 
intrinsic diffusivity matrix) is not possible for one reason or another.

This model predicts that the concentration-based effective binary diffusivity of a component may vary between greater than the intrinsic effective binary diffusivity and smaller than the diffusivity of $\mathrm{SiO}_{2}$ or $\mathrm{SiO}_{2}+\mathrm{Al}_{2} \mathrm{O}_{3}$ and hence may vary significantly if the latter two diffusivities are very different. Concentration-based effective binary diffusivity of a component depends not only on bulk composition but also on compositional gradients of major components and on the component's own concentration gradient, which not only suggests that concentration-based effective binary diffusivities are not very useful but also provides an experimental test for this model. A rigorous test of the model can be carried out if both concentration and activity profiles are measured. The model can be applied semiquantitatively to a variety of geologically interesting diffusion problems, associated with crystal growth, magma mixing, and the evolution of isotopic and concentration profiles.

\section{NOTATION}

A dissolution parameter defined in (15).

a activity of a component in consideration.

C concentration of a component in consideration.

$C_{f} \quad$ hypothetical equilibrium concentration difference of a component between the melt at the original interface and the melt at $\infty$ as defined in (8) or (18); see also Figure 1.

$\left|C_{f}\right| \quad$ absolute value of $C_{f}$.

$C_{\infty}$ initial concentration in the melt (for crystal dissolution).

$C_{s} \quad$ initial concentration in the crystal.

$D$ concentration-based effective binary diffusivity.

$D_{b} \quad \mathrm{D}$ of $\mathrm{SiO}_{2}$ or $\mathrm{SiO}_{2}+\mathrm{Al}_{2} \mathrm{O}_{3}$.

$D_{i j} \quad$ concentration-based cross-term diffusivity of component $i$ due to concentration gradient of component $j$.

[D] concentration-based diffusivity matrix defined in (1).

$\mathcal{D}$ intrinsic effective binary diffusivity.

$\mathcal{D}_{i j} \quad$ intrinsic cross-term diffusivity of component $i$ due to activity gradient of component $j$.

[D] intrinsic diffusivity matrix defined in (3).

$J$ diffusive flux of a component.

UI length of vector $J$.

$\boldsymbol{K}$ two-liquid partition coefficient.

$N$ number of components in a multicomponent system.

$R \quad$ Gas constant.

$T$ temperature (in $\mathrm{K}$ unless otherwise specified).

$t$ time.

$V$ melt growth rate, roughly equals crystal dissolution rate.

$x$ distance (not mole fraction).

$\beta$ a parameter used in (8a) and (18a).

$\Delta a \quad a_{+}-a_{2}$

$\Delta C \quad C_{+}-C_{\text {. }}$.

$\gamma \quad$ activity coefficient of a component.

$\rho \quad$ total mass density.

$\chi^{2} \quad$ chi-square defined in (20).

\section{Subscripts}

e at equilibrium or hypothetical equilibrium.

$i$ component $i$.

$j \quad$ component $j$.

$k$ point $k$

$+\quad$ the $x>0$ side of a diffusion couple.

- the $x<0$ side of a diffusion couple.

Acknowledgments. This work is an outgrowth of my earlier work with D. Walker and C.E. Lesher [Zhang et al., 1989] and I benefited from many discussions with both of them. Discussions with E.M.
Stolper, F.M. Richter, S. van der Laan, and P.D. Ihinger were also very helpful. Helpful reviews and comments were provided by F. Albarede, J. Beckett, S. Chakraborty, A. Cooper, T. Dunn, E. Essene, J. Ganguly, A.C. Lasaga, F.M. Richter, F.J. Spera, A.F. Trial, E.B. Watson, and an anonymous reviewer. The support and encouragement of E.M. Stolper are greatly appreciated.

\section{REFERENCES}

Albarede, F., and Y. Botlinga, Kinetic disequilibrium in trace element partitioning between phenocrysts and host lava, Geochim. Cosmochim. Acta, 36, 141-156, 1972.

Baker, D.R., Tracer vs trace element diffusion: diffusional decoupling of $\mathrm{Sr}$ concentration from $\mathrm{Sr}$ isotope composition, Geochim. Cosmochim. Acta, 53, 3015-3023, 1989.

Baker, D.R., Chemical intendiffusion of dacite and thyolite: anhydrous measurements at $1 \mathrm{~atm}$ and $10 \mathrm{kbar}$, application of transition state theory, and diffusion in zoned magma chambers, Contrib. Mineral. Petrol., 104, 407-423, 1990.

Baker, D.R., Tracer diffusion of network formers and multicomponent diffusion in dacitic and thyolitic melts, Geochim. Cosmochim. Acta, $56,617-632,1992$.

Brearley, M., and C.M. Scarfe, Dissolution rates of upper mantle minerals in an alkali basalt melt at high pressure: an experimental study and implication for ultramafic xenolith survival, J. Petrol., 27, 1157-1182, 1986.

Carman, P.C., Intrinsic mobilities and independent fluxes in multicomponent isothermal diffusion, I, Simple Darken system, II. Complex Darken systems, J. Phys. Chem., 76, 1707-1721, 1968.

Cashman, K.V., Textural constraints on the kinetics of crystallization of igneous rocks, Rev. Mineral., 24, 259-314, 1991.

Cooper, A.R., Model for multi-component diffusion, Phys. Chem. Glasses, 6, 55-61, 1965.

Cooper, A.R., The use and limitations of the concept of an effective binary diffusion coefficient for multi-component diffusion, in Mass Transport in Oxides, edited by J. B. Wachman, and A. D. Franklin, NBS Spec. Publ. 296, 79-84, 1968.

Davidson, J.P., S.L. Desilva, P. Holden, and A.N. Halliday, Smallscale disequilibrium in a magmatic inclusion and its more silicic host, J. Geophys. Res., 95, 17661-17675, 1990.

De Groot, S.R., and P. Mazur, Non-Equilibrium Thermodynamics, 510 pp., Wiley Interscience, New York, 1962.

Donaldson, C.H., The rates of dissolution of olivine, plagioclase, and quartz in a basaltic melt, Mineral. Mag., 49, 683-693, 1985.

Donaldson, C.H., Forsterite dissolution in superheated basaltic, andesitic and thyolitic melts, Mineral. Mag., 54, 67-74, 1990.

Dowty, E., Crystal growth and nucleation theory and the numerical simulation of igneous crystallization, in Physics of Magmatic Processes, edited by R. B. Hargraves, pp. 419-486, Princeton University Press, Princeton, NJ., 1980.

Duda, A., and N. Shimizu, An application of kinetic crystal growth models to trace element zoning in alkali basaltic clinopyroxenes, Mater. Sci. Forwm, 7, 139-144, 1986.

Fisk, M.R., Basaltic magma interaction with harzburgite and the formation of high-magnesium andesites, Geophys. Res. Lett., 13, $467-470,1986$.

Freer, R., Diffusion in silicate minerals and glasses: A data digest and guide to the literature, Contrib. Mineral. Petrol., 76, 440-454, 1981.

Ghiorso, M.S., Chemical mass transfer in magmatic processes, Contrib. Mineral. Petrol., 96, 291-313, 1987a.

Ghiorso, M.S., Modeling magmatic systems: Thermodynamic relations, Rev. Mineral., 17, 443-465, $1987 \mathrm{~b}$.

Ghiorso, M.S., I.S.E. Carmichael, M.L. Rivers, and R.O. Sack, The Gibbs free energy of mixing of natural silicate liquids; an expanded regular solution approximation for the calculation of magmatic intensive variables, Contrib. Mineral. Petrol., 84, 107-145, 1983.

Gupta, P.K., and A.R. Cooper, The [D] matrix for multicomponent diffusion, Physica, 54, 39-59, 1971.

Harrison, T.M., and E.B. Watson, Kinetics of zircon dissolution and zirconium diffusion in granitic melts of variable water content, Contrib. Mineral. Petrol., 84, 66-72, 1983.

Harrison, T.M., and E.B. Watson, The behavior of apatite during crustal anatexis: Equilibrium and kinetic considerations, Geochim. Cosmochim. Acta, 48, 1467-1477, 1984.

Hildreth, W., Gradients in silicic magma chambers: Implications for lithospheric magmatism, J. Geophys. Res., 86, 10,153-10,192, 1981. Hofmann, A.W., Diffusion in natural silicate melts: A critical review, 
in Physics of Magmatic Processes, edited by R.B. Hargraves, pp. 385-417, Princeton University Press, Princeton, N.J., 1980.

Hofmann, A.W., and S.R. Hatt, An assessment of local and regional isotopic equilibrium in the mantle, Earth Planet. Sci. Lett., 38, 44$62,1978$.

Jones, J.H., and D.J. Mavin, A nonmetal interaction model for the segregation of trace metals during solidification of Fe-Ni-S, Fe-Ni-P. and Fe-Ni-S-P alloys, Met. Trans. B, 21B, 697-706, 1990.

Kirkaldy, J.S., and D.J. Young, Diffusion in the Condensed State, 527 pp., Institute of Metals, London, 1987.

Kirkpatrick, R.J., Crystal growth from the melt: A review, Am. Mineral., 60, 798-814, 1975.

Kirkpatrick, R.J., Kinetics of crystallization of igneous rocks, Rev. Mineral., 8, 321-389, 1981.

Kubicki, J.D., G.E. Muncill, and A.C. Lasaga, Chemical diffusion in melts on the $\mathrm{CaMgSi}_{2} \mathrm{O}_{6}-\mathrm{CaAl}_{2} \mathrm{Si}_{2} \mathrm{O}_{8}$ join under high pressures, Geochim. Cosmochim. Acta, 54, 2709-2715, 1990.

Kuo, L.-C., and R.J. Kirkpatrick, Kinetics of crystal dissolution in the system diopside-forsterite-silica, Am.J. Sci., 285, 51-90, 1985.

Lange, R.A., and I.S.E. Carmichael, Densities of $\mathrm{Na}_{2} \mathrm{O}-\mathrm{K}_{2} \mathrm{O}-\mathrm{CaO}$ $\mathrm{MgO}-\mathrm{FeO}-\mathrm{Fe}_{2} \mathrm{O}_{3}-\mathrm{Al}_{2} \mathrm{O}_{3}-\mathrm{TiO}_{2}-\mathrm{SiO}_{2}$ liquids: New measurements and derived partial molar properties, Geochim. Cosmochim. Acta, 51, 2931-2946, 1987.

Lasaga, A.C., Multicomponent exchange and diffusion in silicates, Geochim. Cosmochim. Acta, 43, 455-469, 1979.

Lasaga, A.C., Implication of a concentration dependent growth rate on the boundary layer crystal-melt model, Earth Planet. Sci. Lett., 56 , 429-434, 1981.

Lasaga A.C., Toward a master equation in crystal growth, Am. J. Sci., 282, 1264-1288, 1982.

Lesher, C.E., Effects of silicate liquid composition on mineral-liquid element partitioning from Soret diffusion studies, J. Geophys. Res., 91,6123-6141, 1986 .

Lesher, C.E., Decoupling of chemical and isotopic exchange during magma mixing, Nature, 344, 235-237, 1990.

Lesher, C.E., Chemical and self diffusivities of $\mathrm{Sr}$ and $\mathrm{Nd}$ in naturallyoccurring silicate liquids, Eos Trans. AGU, 72(17), Spring Meeting Suppl., 309, 1991.

McKenzie, D., The generation and compaction of partially molten rock, J. Petrol., 25, 713-765, 1984.

Press, W.H., B.P. Flannery, S.A. Teukolsky, and W.T. Vetterling, Numerical Recipes, 702 pp., Cambridge University Press, New York, 1989.

Richter, F.M., and E.M. Stolper, A method for determining chemical activities using multi-component diffusion experiments, Eos Trans. $A G U, 72(44)$, Fall Meeting Suppl., 528, 1991.

Ryerson, F.J., and P.C. Hess, Implications of liquid-liquid distribution coefficients to mineral-liquid partitioning, Geochim. Cosmochim. Acto, 42, 921-932, 1978.
Ryerson, F.J., and E.B. Watson, Rutile saturation in magmas: Implication for Ti-Nb-Ta depletion in island-arc basalts, Earth Planet. Sci. Lett., 86, 225-239, 1987.

Sato, H., Diffusion coronas around quartz xenocrysts in andesite and basalt from Tertiary volcanic region in northeastem Shikoku, Japan, Contrib. Mineral. Petrol., S0, 46-64, 1975.

Shewmon, P.G., Diffusion in Solids, 203 pp., McGraw-Hill, New York, 1963.

Sneeringer, M., S.R. Hart, and N. Shimizu, Strontium and samarium diffusion in diopside, Geochim. Cosmochim. Acta, 48, 1589-1608, 1984.

Spera, F.J., and A.F. Trial, Verification of Onsager's reciprocal relations in a molten silicate solution, Science, 259, 204-206, 1993.

Spera, F.J., C.M. Oldenburg, and D.A. Yuen, Magma zonation: Effects of chemical buoyancy and diffusion, Geophys. Res. Lett., 16, 1387 $1390,1989$.

Trial, A.F., and F.J. Spera, Natural convection boundary layer flows in isothermal temary systems: role of diffusive coupling, Int. J. Heat Transfer, 31, 941-955, 1988.

Trial, A.F., and F.J. Spera, Multicomponent diffusion matrix calculated from experimental rhyolite-dacite interdiffusion data, Eos Trans. $A G U, 71,1163,1990$

Tsuchiyama, A., Dissolution kinetics of plagioclase in the melt system of diopside-albite-anorthite and origin of dusty plagioclase in andesites, Contrib. Mineral. Petrol., 89, 1-16, 1985.

Tsuchiyama, A., and E. Takahashi, Melting kinetics of a plagioclase feldspar, Contrib. Mineral. Petrol., 84, 345-354, 1983.

van der Laan, S.R., A. Kennedy, G.J. Wasserburg, P.J. Wylie, and Y. Zhang, Preliminary comparison of chemical and self diffusion for $K$ and $\mathrm{Ca}$ in andesite and rhyolite melt, Eos Trans. AGU, 71, 652, 1990.

Watson, E.B., Two-liquid partitioning coefficients: experimental data and geochemical implications, Contrib. Mineral. Petrol., 56, 119 134, 1976.

Watson, E.B., Basalt contamination by continental crust: Some experiments and models, Contrib. Mineral. Petrol., 80, 73-87, 1982.

Watson, E.B., and D.R. Baker, Chemical diffusion in magmas: an overview of experimental results and geochemical implications, in Adv. Phys. Geochem., 6, edited by I. Kushiro, and L. Perchuk, PP. 120-151, 1991.

Zhang, Y., D. Wlaker, and C.E. Lesher, Diffusive crystal dissolution, Contrib. Mineral. Petrol., 102, 492-513, 1989.

Y. Zhang, Department of Geological Sciences, University of Michigan, Ann Arbor, MI 48109-1063.

(Received September 30, 1992; revised February 1, 1993; accepted February 18, 1993.) 